Karl A. Kalina, Alexander Raßloff, Maximilian Wollner, Philipp Metsch, Jörg Brummund and Markus Kästner*

\title{
24 Multiscale modeling and simulation of magneto-active elastomers based on experimental data
}

\begin{abstract}
In this contribution, we present a framework for the multiscale modeling and simulation of magneto-active elastomers (MAEs). It enables us to consider these materials on the microscopic scale, where the heterogeneous microstructure consisting of magnetizable particles and elastomer matrix is explicitly resolved, as well as the macroscopic scale, where the MAE is considered to be a homogeneous magneto-active body. On both scales, a general continuum formulation of the coupled magneto-mechanical boundary value problem is applied and the finite element method is used to solve the governing equations. Starting with an experimental characterization of the individual constituents, i.e. particles and matrix, microscopic constitutive models for both are formulated and adjusted to the experimental data separately. With that, properties of MAEs resulting from the microscopic constitutive behavior can be captured within the presented modeling approach. Secondly, to discuss general macroscopic properties of magnetically soft and hard MAEs, a computational homogenization scheme is used to calculate the composites' effective behavior for different geometrical arrangements of the particles on the microscale. Finally, the calculated effective response of a magnetically soft composite system is used to identify the parameters of a macroscopic magneto-elastic model. Using the calibrated model, the behavior of macroscopic MAEs is simulated for different sample geometries.
\end{abstract}

Keywords: finite element simulation, magneto-active elastomers, magneto-mechanics, multiscale

Mathematics Subject Classification 2010: 65C05, 62M20, 93E11, 62F15, 86A22

\footnotetext{
*Corresponding author: Markus Kästner, Chair of Computational and Experimental Solid Mechanics, Institute of Soild Mechanics, TU Dresden, 01062 Dresden, Germany, E-mail: markus.kaestner@tudresden.de

Karl A. Kalina, Alexander Raßloff, Maximilian Wollner, Philipp Metsch and Jörg Brummund, Chair of Computational and Experimental Solid Mechanics, Institute of Soild Mechanics, TU Dresden, 01062 Dresden, Germany

O Open Access. (c) 2020 Karl A. Kalina et al., published by De Gruyter. (c) BY-NC-ND This work is licensed under the Creative Commons Attribution-NonCommercial-NoDerivatives 4.0 International License.

This article has previously been published in the journal Physical Sciences Reviews. Please cite as: K. A. Kalina, A. Raßloff, M. Wollner, P. Metsch, J. Brummund and M. Kästner, "Multiscale modeling and simulation of magneto-active elastomers based on experimental data" Physical Sciences Reviews [Online] 2020, 5. DOI: 10.1515/psr-2020-0012 | https://doi.org/10.1515/ 9783110569636-024
} 


\subsection{Introduction}

Magneto-active elastomers (MAEs) are a special class of composite materials that alter their effective macroscopic behavior if an external magnetic field is applied. Due to the strong coupling behavior, they are attractive for several engineering applications such as actuators and sensors [1-4], valves [5], tunable vibration absorbers [6], medical robots [7] or prosthetic and orthotic devices with controllable stiffness [8].

Basically, MAEs consist of a soft polymer matrix filled with micron-sized magnetizable particles that can be arranged in an unstructured distribution or, if external fields are applied during the manufacturing process, in a chain-like [9-11] or even more complex structure [12]. Regarding the resulting macroscopic behavior, this leads to isotropic and anisotropic properties, respectively. Besides the underlying particle distribution, the effective magneto-mechanical behavior of the MAE will depend on the properties of the individual constituents. If the elastomer is combined with magnetically soft fillers as carbonyl iron, a reversible behavior will be observed. In contrast to that, a strongly irreversible behavior results if magnetically hard fillers, e.g. NdFeB or $\mathrm{CoFe}_{2} \mathrm{O}_{4}$, with strong magnetic hysteresis are used [13-17].

\subsubsection{Modeling approaches}

Strategies regarding the modeling of MAEs can be divided into microscopic and macroscopic approaches. In the former, the heterogeneous microstructure consisting of particles and matrix is explicitly resolved whereas the composite is regarded as macroscopically homogeneous in the latter, see Figure 24.1(a).

(a)

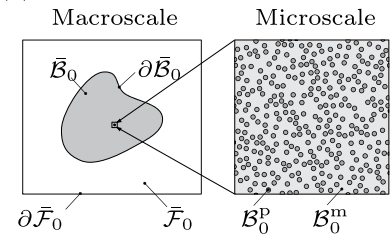

(b)

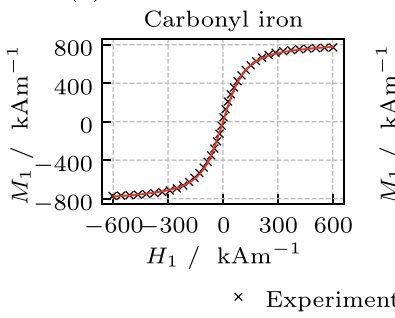

(c)

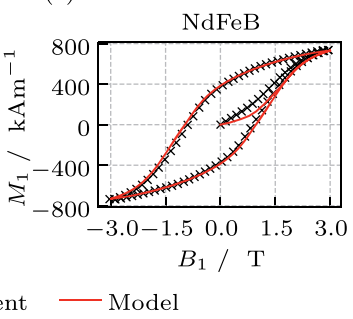

Figure 24.1: Continuum based modeling of MAEs: (a) macroscopic sample $\overline{\mathcal{B}}_{0}$ embedded into the free space $\overline{\mathcal{F}}_{0}$ and underlying heterogeneous microstructure consisting of magnetizable particles $\mathcal{B}_{0}^{\text {p }}$ and surrounding elastomer matrix $\mathcal{B}_{0}^{\mathrm{m}}$, and (b), (c) measured magnetization curve and adjusted model (25) for magnetically soft carbonyl iron and major hysteresis loop of magnetically hard NdFeB particles and parameterized model (26)-(29). Experimental data given in (b) and (c) are taken from Spieler et al. [18] and Linke et al. [14], respectively. 


\section{Microscopic approaches}

The first class of microscopic approaches is represented by particle-interaction models which are based on an energy minimization and the assumption, that the particles are described by magnetic dipoles [19-21]. Within these models, the polymer matrix is considered to be a continuous elastic medium or it is represented by springs between the particles, see Menzel [22] or Menzel and Löwen [23]. Particle-interaction models are numerically quite efficient but, due to the dipole assumption, their usage is limited to dilute systems. An extension of particle-interaction models to tightly filled systems is shown by Biller et al. [24], where a multipole expansion is used to calculate the particles' mutual magnetic interactions.

The second class of microscopically motivated approaches are continuum based models which resolve the local magnetic as well as mechanical fields within the composite [13, 25-30]. Thus, these approaches are not limited to the modeling of systems with a low amount of magnetic filler particles. Furthermore, they can be adapted to several kinds of dissipative materials, easily [13]. Microscopic continuum approaches also offer the possibility to analyze the stability of magneto-active materials under different loading conditions [31, 32]. However, the computationally expensive numerical solution of the underlying magneto-mechanical boundary value problem (BVP) which is usually done with the finite element (FE) method is a clear disadvantage of these models: in order to predict the effective material behavior of realistic magneto-active composites, an appropriate homogenization scheme as introduced by Chatzigeorgiou et al. [33] has to be used. A tool to capture not only the microstructure but also the shape of the macroscopic samples is the $\mathrm{FE}^{2}$ method as performed by Keip and Rambausek [34, 35].

\section{Macroscopic approaches}

Macroscopic models represent an entirely different approach for the description of MAEs. Since the microstructure is not explicitly resolved and the composite is considered as a homogeneous continuum, they enable the representation of real structures with a reasonable computational effort. To capture the effects of the underlying microstructure implicitly, magneto-mechanical coupling terms have to be incorporated in these models. A variety of macroscopic approaches for the isotropic and transversely isotropic magneto-elastic case [9, 36-38] or the rate-dependent magneto-viscoelastic case [39-41] can be found in the literature.

Naturally, all of these models are purely phenomenological, i.e. fitting parameters have to be determined. This is done based on experimental results $[9,41]$ or by using data generated from a microscopic model combined with analytical [42, 43] or computational homogenization approaches [44]. If the fitting is done with experimental data, it is important to note, that at least inhomogeneous mechanical fields occur independent of the macroscopic sample geometry [35, 44, 45]. Moreover, if no ellipsoidal samples $[46,47]$ are used within the experiments, also the magnetization is 
inhomogeneous, e.g. in the case of widely used cylindrical shaped specimens. Due to this, the parameterized macroscopic model contains the influence of the sample geometry, i.e. the model describes the behavior of the MAE-sample and not the constitutive behavior of the pure MAE composite material. The fitting based on data generated from microscopic models avoids the described difficulty since it allows the identification of the parameters independent of any macroscopic sample geometry.

\subsubsection{Content}

In this contribution, a multiscale modeling approach based on Metsch et al. [29,30] and Kalina et al. $[13,28,44]$ is presented. Results of these works are summarized and partially supplemented in the present article. Within the developed multiscale modeling framework, MAEs are described based on a general continuum formulation, where the governing equations are solved with the FE method.

In order to achieve a deep understanding of the MAEs' effective behavior, the constitutive properties of the individual phases on the microscale as well as their interactions have to be investigated. Starting with an experimental characterization of the individual constituents, microscopic constitutive models are formulated and fitted to the measured data. This includes models for magnetically hard and soft filler particles and an adjusted hyperelastic model for a specific silicone elastomer. To discuss general macroscopic properties of magnetically soft and hard MAEs in a second step, computational homogenizations are preformed to calculate the composites' effective behavior for different microscopic particle arrangements. The approach is usable for the $2 \mathrm{D}$ and the 3D case, where both cases are compared. Finally, a macroscopic isotropic model for magnetically soft MAEs is parameterized by using homogenized data. It is used to analyze the behavior of MAEs for several sample geometries.

The organization of the paper is as follows: In Section 24.2, a general magnetomechanical continuum formulation and the applied numerical framework are given. The microscopic constitutive models including their parametrization are described in Section 24.3. In Section 24.4, the multiscale approach is applied to study the behavior of several microstructures and in Section 24.5, a macroscopic model and its parametrization is shown. After a discussion of the results, the paper is closed by concluding remarks and an outlook to necessary future work.

\subsection{Theoretical framework}

In this section, the basic equations of the coupled magneto-mechanical BVP are summarized. This includes the field equations, general constitutive relations and a scale transition scheme which connects the micro- and the macroscale. Furthermore, 
an FE formulation, which is necessary for the numerical solution, is introduced. The theoretical framework is given in a condensed form. For more details, the reader is referred to the works [13, 28-30, 44].

\subsubsection{Continuum formulation}

According to the basic concept of continuum mechanics, a material body occupies the current and the reference configurations $\mathcal{B} \subset \mathcal{R}^{3}$ and $\mathcal{B}_{0} \subset \mathcal{R}^{3}$ with boundaries $\partial \mathcal{B}$ and $\partial \mathcal{B}_{0}$, respectively. In the following, associated Eulerian and Lagrangian fields are denoted by lower- and uppercase letters. Vectors and higher-order tensors are indicated by boldface italic characters. The norms of vectors and second order tensors are written as $|\boldsymbol{v}|:=\sqrt{v_{l} v_{l}}$ and $\|\boldsymbol{T}\|:=\sqrt{T_{k l} T_{k l}}$, respectively. Therein, the tensor coordinates are given with respect to a Cartesian coordinate system and the Einstein summation convention is applied. Furthermore, the symbols $\nabla$ and $\nabla_{\boldsymbol{X}}$ denote partial derivatives of a quantity $(\bullet)$ with respect to the spatial and reference configurations. Derivatives with respect to a tensor quantity $\boldsymbol{t}$ are given by $\partial_{\boldsymbol{t}}(\bullet)$ within the running text. Finally, the jump across material surfaces of discontinuity $\mathcal{S}$ and $\mathcal{S}_{0}$, with unit normal vectors $\boldsymbol{n}$ and $\boldsymbol{N}$ pointing from subdomains $\mathcal{B}^{-}$to $\mathcal{B}^{+}$and $\mathcal{B}_{0}^{-}$to $\mathcal{B}_{0}^{+}$, is denoted by $\llbracket(\bullet) \rrbracket=(\bullet)^{+}-(\bullet)^{-}$.

\subsubsection{Field equations}

Regarding the MAEs' microstructure, the embedded magnetizable particles are multidomain particles with a mean diameter of several micro meters, which is considerably larger than the polymer chain length. Thus, the assumption of a continuum and the following field equations are valid on the both considered scales of the material.

\section{Kinematics}

The first set of equations is given by kinematic relations. Each material point of the considered body is identified by its position vector $\boldsymbol{X} \in \mathcal{B}_{0}$ at time $t=t_{0}$. Due to the displacement $\boldsymbol{u}(\boldsymbol{X}, t):=\boldsymbol{\varphi}(\boldsymbol{X}, t)-\boldsymbol{X}$ of these points, the actual position changes to $\boldsymbol{x} \in \mathcal{B}$ at $t>t_{0}$. Therein, $\boldsymbol{\varphi}$ is a mapping function that is continuous in space and time, i.e. $\llbracket \boldsymbol{\varphi} \rrbracket=\mathbf{0}$ holds on $\mathcal{S}$. The deformation gradient $\boldsymbol{F}$ and its determinant $J$ are defined by the relations

$$
\boldsymbol{F}:=\left(\nabla_{\boldsymbol{X}} \boldsymbol{\varphi}\right)^{\mathrm{T}} \text { and } J:=\operatorname{det}(\boldsymbol{F})>0 .
$$

Finally, the symmetric right Cauchy-Green deformation tensor $\boldsymbol{C}:=\boldsymbol{F}^{\mathrm{T}} \cdot \boldsymbol{F}$ is introduced as a further kinematic quantity which is free of rigid body motions. 


\section{Maxwell equations and mechanical balance laws}

The second set of relations are the two stationary magnetic Maxwell equations and the mechanical balance laws. For vanishing current densities $\boldsymbol{j}$ as well as neglected mechanical body forces $\varrho f$ and inertia terms $\varrho \ddot{x}$, these equations and corresponding jump conditions are given by

$$
\begin{aligned}
\nabla \cdot \boldsymbol{b} & =0 \text { with } \boldsymbol{n} \cdot \llbracket \boldsymbol{b} \rrbracket=0 \quad \text { on } \mathcal{S}, \\
\nabla \times \boldsymbol{h} & =\mathbf{0} \quad \text { with } \quad \boldsymbol{n} \times \llbracket \boldsymbol{h} \rrbracket=\mathbf{0} \quad \text { on } \mathcal{S}, \\
J \varrho-\varrho_{0} & =0, \\
\nabla \cdot \boldsymbol{\sigma}^{\text {tot }} & =\mathbf{0} \text { with } \boldsymbol{n} \cdot \llbracket \boldsymbol{\sigma}^{\text {tot }} \rrbracket=-\widehat{\boldsymbol{t}} \quad \text { on } \mathcal{S} \text { and } \\
\text { skw } \boldsymbol{\sigma}^{\text {tot }} & =\mathbf{0}
\end{aligned}
$$

with respect to the current configuration $\mathcal{B}$ [48-50]. In the equations above, $\boldsymbol{b}$ and $\boldsymbol{h}$ denote the magnetic induction and field which are linked by the permeability of free space $\mu_{0}$ and the magnetization $\boldsymbol{m}$ via the equation

$$
\boldsymbol{b}=\mu_{0}(\boldsymbol{h}+\boldsymbol{m}) .
$$

The quantities $\varrho$ and $\varrho_{0}$ denote the mass density with respect to $\mathcal{B}$ and $\mathcal{B}_{0}$, respectively. Furthermore, the symbols $\boldsymbol{\sigma}^{\text {tot }}$ and $\hat{\boldsymbol{t}}$ indicate the total Cauchy stress tensor and a mechanical traction vector on $\mathcal{S}$, where $\boldsymbol{\sigma}^{\text {tot }}$ is given as the sum of the mechanical and magnetic stress tensors [28-30, 49].

Besides the introduced Eulerian quantities, Lagrangian fields related to $\mathcal{B}_{0}$ are defined. With them, an objective formulation of constitutive models is ensured. Furthermore, they are necessary for a total-Lagrangian FE formulation [28], which is applied here. The Lagrangian magnetic quantities $\boldsymbol{B}, \boldsymbol{H}$ and $\boldsymbol{M}$ as well as the total first Piola-Kirchhoff stress tensor $\boldsymbol{P}^{\text {tot }}$ and the corresponding mechanical traction vector $\hat{\boldsymbol{p}}$ are obtained from the pullback operations [28]

$$
\begin{aligned}
\boldsymbol{B} & :=J \boldsymbol{F}^{-1} \cdot \boldsymbol{b}, \quad \boldsymbol{H}:=\boldsymbol{F}^{\mathrm{T}} \cdot \boldsymbol{h} \text { and } \boldsymbol{M}:=\boldsymbol{F}^{\mathrm{T}} \cdot \boldsymbol{m} \text { as well as } \\
\boldsymbol{P}^{\mathrm{tot}} & :=J \boldsymbol{F}^{-1} \cdot \boldsymbol{\sigma}^{\mathrm{tot}} \text { and } \hat{\boldsymbol{p}}:=J\left|\boldsymbol{F}^{-\mathrm{T}} \cdot \boldsymbol{N}\right| \widehat{\boldsymbol{t}} .
\end{aligned}
$$

It should be noted, that the introduced operations are not unique, but the choice according to Eqs. (24.8) and (24.9) leads to a preserved structure of the Eqs. (24.2)-(24.5) in their Lagrangian form $[36,38]$, e.g. $\nabla_{\boldsymbol{X}} \cdot \boldsymbol{B}=0$.

In order to reduce the set of equations that has to be solved, it is favorable to introduce a magnetic potential. If, for example, the magnetic scalar potential $\varphi$ which is defined by $\boldsymbol{H}=:-\nabla_{X} \varphi$ is used, Eq. (24.3) is satisfied automatically and only Eq. (24.2) remains to solve the magnetic part of the BVP. Likewise, the magnetic vector potential $\boldsymbol{A}$ which is defined by the relation $\boldsymbol{B}=: \nabla_{\boldsymbol{X}} \times \boldsymbol{A}$ is usable. In contradiction to $\varphi$, now Eq. (24.2) is satisfied automatically and Eq. (24.3) has to be solved. A common way to ensure 
uniqueness of $\boldsymbol{A}$, is to demand the satisfaction of the Coulomb gauge $\nabla_{\boldsymbol{X}} \cdot \boldsymbol{A}=0$. Note that this equation is fulfilled automatically in the $2 \mathrm{D}$ case but needs to be considered as a constraint in the 3D case [51].

\section{Constitutive equations}

Finally, the set of field equations is completed by the constitutive relations. In order to ensure thermodynamic consistency, the second law of thermodynamics has to be fulfilled for all values of the independent constitutive variables and their rates at any time $t$. Thus, the Clausius-Duhem inequality (CDI), which is derived from the balance of internal energy, the entropy balance, the second law of thermodynamics as well as the previously introduced balance equations has to be evaluated [49]. Assuming temperature fields that are homogeneous in space and time, the CDI in its Lagrangian form reduces to

$$
-\dot{\Omega}+\boldsymbol{H} \cdot \dot{\boldsymbol{B}}+\boldsymbol{P}^{\mathrm{tot}}: \dot{\boldsymbol{F}} \geq 0 \quad \text { with } \Omega:=\varrho_{0} \Psi\left(\boldsymbol{C}, \boldsymbol{B}, \boldsymbol{Q}^{\alpha}, \boldsymbol{Z}^{\beta}\right)+\frac{1}{2 \mu_{0} J} \boldsymbol{C}:(\boldsymbol{B} \otimes \boldsymbol{B})
$$

in the magneto-mechanical case. Therein, $\Omega$ denotes the so called amended free energy density function [36] which is decomposed into the specific Helmholtz free energy $\Psi$, that depends on $\boldsymbol{C}$ and $\boldsymbol{B}$ as well as possible vector or tensor-valued internal variables $\boldsymbol{Q}^{\alpha}, \boldsymbol{Z}^{\alpha}$ and a material independent free space part which follows from the pull back of the magnetic free field energy $\mu_{0}^{-1} \boldsymbol{b} \cdot \boldsymbol{b}$. Following the procedure of Coleman and Noll [52], the constitutive relations

$$
\boldsymbol{P}^{\mathrm{tot}}=2 \frac{\partial \Omega}{\partial \boldsymbol{C}} \cdot \boldsymbol{F}^{\mathrm{T}}, \quad \boldsymbol{H}=\frac{\partial \Omega}{\partial \boldsymbol{B}} \text { and }-\frac{\partial \Omega}{\partial \boldsymbol{Q}^{\alpha}} \cdot \dot{\boldsymbol{Q}}^{\alpha}-\frac{\partial \Omega}{\partial \boldsymbol{Z}^{\alpha}}: \dot{\boldsymbol{Z}}^{\alpha} \geq 0
$$

result from the evaluation of (24.10). The described formulation is suitable if the magnetic vector potential $\boldsymbol{A}$ is used, since $\boldsymbol{B}$ - which serves as independent constitutive variable in $\mathbf{\Omega}$ - could be derived directly from $\boldsymbol{A}$.

If instead a magnetic scalar potential formulation is chosen, it is favorable to use $\boldsymbol{H}$ instead of $\boldsymbol{B}$ as an independent constitutive variable. Therefore, a further amended free energy density function $\Omega^{\star}$ is introduced by the Legendre-Fenchel transformation

$$
\begin{aligned}
\Omega^{\star}\left(\boldsymbol{C}, \boldsymbol{H}, \boldsymbol{Q}^{\alpha}, \boldsymbol{Z}^{\beta}\right) & :=\inf _{\boldsymbol{B}}\left\{\Omega\left(\boldsymbol{C}, \boldsymbol{B}, \boldsymbol{Q}^{\alpha}, \boldsymbol{Z}^{\beta}\right)-\boldsymbol{H} \cdot \boldsymbol{B}\right\} \\
& =\varrho_{0} \Psi^{\star}\left(\boldsymbol{C}, \boldsymbol{H}, \boldsymbol{Q}^{\alpha}, \boldsymbol{Z}^{\beta}\right)-\frac{\mu_{0}}{2} J \boldsymbol{C}^{-1}:(\boldsymbol{H} \otimes \boldsymbol{H}) .
\end{aligned}
$$

Regarding Eq. (24.11), the second relation is now replaced by $\boldsymbol{B}=-\partial_{\boldsymbol{H}} \Omega^{\star}$, where the other relations stay unchanged. As already stated, the presented set of equations holds on the micro- and the macroscale. The representation of the individual properties of the materials is inserted via a suitable choice of the free energy functions $\Psi$ or $\Psi^{\star}$. 


\subsubsection{Scale transition scheme}

To connect microscopic and macroscopic quantities, a magneto-mechanical homogenization scheme is used. Depending on the choice of the independent variables, i.e. $\{\boldsymbol{u}, \boldsymbol{A}\}$ or $\{\boldsymbol{u}, \varphi\}$, the scale transition scheme has to be adjusted. In this work, the set of equations is exemplarily given for the scalar potential formulation. The reader is referred to Metsch et al. [29] or Kalina et al. [13] for the case of a vector potential formulation.

An effective macroscopic quantity $(\cdot \vec{\bullet})$, that is related to the macroscale, is identified from the microscopic field distribution within a representative volume element (RVE) by the volume average $\langle(\bullet)\rangle$. To ensure a physically meaningful scale transition, the equivalence of the macroscopic and the averaged microscopic energies which is also known as the Hill-Mandel condition [53] has to be fulfilled. In the magnetomechanical case this relation is given by

$$
\left\langle\boldsymbol{P}^{\text {tot }}: \dot{\boldsymbol{F}}\right\rangle-\langle\boldsymbol{B} \cdot \dot{\boldsymbol{H}}\rangle=\overline{\boldsymbol{P}}^{\text {tot }}: \dot{\overline{\boldsymbol{F}}}-\overline{\boldsymbol{B}} \cdot \dot{\overline{\boldsymbol{H}}}
$$

This condition is fulfilled if, for instance, periodic displacement and potential spaces in combination with antiperiodic fluxes are chosen:

$$
\begin{aligned}
\mathcal{U}(\overline{\boldsymbol{F}}) & :=\left\{\boldsymbol{u} \in \mathcal{R}^{d} \mid \boldsymbol{u}=(\overline{\boldsymbol{F}}-\boldsymbol{I}) \cdot \boldsymbol{X}+\tilde{\boldsymbol{u}} \text { with } \tilde{\boldsymbol{u}}^{+}=\tilde{\boldsymbol{u}}^{-}\right\}, \\
\mathcal{P}(\overline{\boldsymbol{H}}) & :=\left\{\varphi \in \mathcal{R} \mid \varphi=-\overline{\boldsymbol{H}} \cdot \boldsymbol{X}+\tilde{\varphi} \text { with } \tilde{\varphi}^{+}=\tilde{\varphi}^{-}\right\} \text {and } \\
\left(\boldsymbol{N} \cdot \boldsymbol{P}^{\mathrm{tot}}\right)^{+} & =-\left(\boldsymbol{N} \cdot \boldsymbol{P}^{\mathrm{tot}}\right)^{-} \text {and }(\boldsymbol{N} \cdot \boldsymbol{B})^{+}=-(\boldsymbol{N} \cdot \boldsymbol{B})^{-} .
\end{aligned}
$$

In Eqs. (24.14)-(24.16), $(\bullet)^{+}$and $(\bullet)^{-}$are values on associated opposing boundaries $\partial \mathcal{B}_{0}^{\alpha+}$ and $\partial \mathcal{B}_{0}^{\alpha-}, \alpha \in\{1, \ldots, d\}$ of the RVE with the dimension $d$. The symbol ( $\left.\widetilde{\bullet}\right)$ marks a fluctuation quantity.

\subsubsection{Numerical solution}

The introduced nonlinear magneto-mechanical BVP is solved by using a total Lagrangian FE formulation. As in the previous subsection, the general equations are exemplarily given for the case of a scalar potential formulation. It is based on the weak forms

$$
\begin{aligned}
& 0=\int_{\mathcal{B}_{0}} \boldsymbol{P}^{\mathrm{tot}}: \delta \boldsymbol{F} \mathrm{d} V_{0}-\oint_{\partial \mathcal{B}_{0}} \hat{\boldsymbol{p}}^{\mathrm{tot}} \cdot \delta \boldsymbol{u} \mathrm{d} S_{0} \text { and } \\
& 0=\int_{\mathcal{B}_{0}} \boldsymbol{B} \cdot \delta \boldsymbol{H} \mathrm{d} V_{0}+\oint_{\partial \mathcal{B}_{0}} \hat{\eta} \delta \varphi \mathrm{d} S_{0}
\end{aligned}
$$


of the Lagrangian versions of Eqs. (24.2) and (24.5), where $\delta \boldsymbol{u}$ and $\delta \varphi$ denote the virtual displacement and scalar potential which have to vanish on the essential boundaries $\partial \mathcal{B}_{0}^{u}$ and $\partial \mathcal{B}_{0}^{\varphi}$, respectively [30]. The virtual deformation gradient $\delta \boldsymbol{F}$ and the virtual Lagrangian magnetic field $\delta \boldsymbol{H}$ are defined by $\delta \boldsymbol{F}:=\left(\nabla_{\boldsymbol{X}} \delta \boldsymbol{u}\right)^{\mathrm{T}}$ and $\delta \boldsymbol{H}:=-\nabla_{\boldsymbol{X}} \delta \varphi$, respectively. Furthermore, $\hat{\boldsymbol{p}}^{\text {tot }}$ and $\hat{\eta}$ denote prescribed nominal stress vectors including magnetic and mechanical loads on $\partial \mathcal{B}_{0}^{p}$ and fluxes $\boldsymbol{B} \cdot \boldsymbol{N}$ on $\partial \mathcal{B}_{0}^{\eta}$, respectively.

The weak forms according to Eqs. (24.17) and (24.18) are solved numerically by approximating the configuration $\mathcal{B}_{0}$ with $n^{\text {el }}$ finite elements capturing the domains $\mathcal{B}_{0}^{e}$, in which the independent primary fields $\{\boldsymbol{u}, \varphi\}$ and their virtual counterparts $\{\delta \boldsymbol{u}, \delta \varphi\}$ are approximated with the shape functions $N^{\alpha}$. Here, $\alpha$ denotes the global node number. Due to the arbitrariness of $\delta \boldsymbol{u}$ and $\delta \varphi$, the mechanical and magnetic residues

$$
\begin{aligned}
\mathbf{R}^{\alpha} & :=\bigcup_{e=1}^{n^{\mathrm{el}}}\left[\int_{\mathcal{B}_{0}^{e}} \nabla_{\boldsymbol{X}} N^{\alpha} \cdot \boldsymbol{P}^{\mathrm{tot}} \mathrm{d} V_{0}-\int_{\partial \mathcal{B}_{0}^{p, e}} N^{\alpha} \widehat{\boldsymbol{p}}^{\mathrm{tot}} \mathrm{d} S_{0}\right]=0 \text { and } \\
\mathrm{R}^{\alpha} & :=\bigcup_{e=1}^{n^{\mathrm{el}}}\left[\int_{\mathcal{B}_{0}^{e}} \nabla_{\boldsymbol{X}} N^{\alpha} \cdot \boldsymbol{B} \mathrm{d} V_{0}-\int_{\partial \mathcal{B}_{0}^{\eta, e}} N^{\alpha} \hat{\eta} \mathrm{d} S_{0}\right]=0
\end{aligned}
$$

follow from the weak forms given in Eqs. (24.17) and (24.18). Within the FE code, the set of coupled nonlinear Eqs. (24.19) and (24.20) is solved by applying a Newton-Raphson scheme at each time step $t_{n}$. To this end, the linear algebraic system

$$
\left[\begin{array}{ll}
\mathbf{K}_{u u}^{\alpha \beta} & \mathbf{K}_{u \varphi}^{\alpha \beta} \\
\mathbf{K}_{\varphi u}^{\alpha \beta} & \mathbf{K}_{\varphi \varphi}^{\alpha \beta}
\end{array}\right]\left[\begin{array}{c}
\Delta \boldsymbol{u}^{\beta} \\
\Delta \varphi^{\beta}
\end{array}\right]=-\left[\begin{array}{c}
\mathbf{R}^{\alpha} \\
\mathbf{R}^{\alpha}
\end{array}\right] \text { with } \alpha, \beta \in\left\{1,2, \ldots, n^{\text {nd }}\right\}
$$

has to be solved for the total number of global nodes $n$ nd in each iteration. Therein, $\mathbf{K}_{x y}^{\alpha \beta}$ with $x, y$ being $u$ or $\varphi$, denote the tangent terms which follow from Gâteaux-derivations of $\mathbf{R}^{\alpha}$ and $\mathrm{R}^{\alpha}$ with respect to $\Delta \boldsymbol{u}$ and $\Delta \varphi$, respectively [28,30]. If a homogenization problem according to the subsection 24.2.1.2 is solved, the primary fields $\boldsymbol{u}$ and $\varphi$ and thus the nodal values $\boldsymbol{u}^{\alpha}$ and $\varphi^{\alpha}$ have to be elements of the spaces $\mathcal{U}(\overline{\boldsymbol{F}})$ and $\mathcal{P}(\overline{\boldsymbol{H}})$ according to (24.14) and (24.15).

All simulations were performed within a MATLAB-based in-house FE framework using the PARDISO solver project $[54,55]$ or, if large 3D problems are considered, the software tool FEniCS [56, 57].

\subsection{Characterization of the constituents}

As shown in Figure 24.1(a), the MAEs' heterogeneous microstructure consists of subdomains $\mathcal{B}_{0}^{\mathrm{p}}$ and $\mathcal{B}_{0}^{\mathrm{m}}$ denoting the particle and the matrix material phases. In the following section, the behavior of these individual constituents is characterized by 
using experimental results. Based on this, constitutive models which describe the behavior of an MAE at the microscopic level are given for both phases.

\subsubsection{Particles}

The magnetizable particles are very stiff compared to the soft elastomer matrix, so that $\boldsymbol{C} \approx \boldsymbol{I}$ holds in $\mathcal{B}_{0}^{\mathrm{p}}$. Therefore, the magneto-mechanical coupling effects in the particles are negligible and the free energy functions introduced in Eqs. (24.10) and (24.12) could be divided into a purely mechanical part and a purely magnetic part [13, $27,28,58$, 59], i.e.

$$
\begin{aligned}
\Psi\left(\boldsymbol{C}, \boldsymbol{B}, \boldsymbol{Q}^{\alpha}\right) & :=\Psi^{\text {mech }}(\boldsymbol{C})+\Psi^{\mathrm{mag}}\left(\boldsymbol{B}, \boldsymbol{Q}^{\alpha}\right) \text { or } \\
\Psi^{\star}\left(\boldsymbol{C}, \boldsymbol{H}, \boldsymbol{Q}^{\alpha}\right) & :=\Psi^{* \text { mech }}(\boldsymbol{C})+\Psi^{* \operatorname{mag}}\left(\boldsymbol{H}, \boldsymbol{Q}^{\alpha}\right) .
\end{aligned}
$$

Independent of the magnetic properties of the particles, the mechanical part is described by a compressible neo-Hookean potential

$$
\Psi^{\text {mech }}\left(I_{1}, J\right):=\frac{1}{2 \rho_{0}}\left[\mu\left(I_{1}-\ln J^{2}-3\right)+\frac{\lambda}{2}\left(J^{2}-\ln J^{2}-1\right)\right]
$$

with $I_{1}:=\operatorname{tr} \boldsymbol{C}$ and $\lambda$ and $\mu$ denoting the Lamé parameters. The particles stiffness should be chosen such that it is large compared to the one of the matrix. With that, they are represented as nearly rigid in the FE calculation. The description of the magnetic part depends on the properties of the particles, where a distinction between magnetically soft and hard behavior is done in the following.

\subsubsection{Magnetically soft particles}

Since magnetically soft materials do not exhibit a load dependent behavior, the actual state of the material only depends on the chosen primary magnetic quantity, i.e. $\boldsymbol{B}$ or $\boldsymbol{H}$. Exemplarily, a suitable description is shown for a scalar potential formulation in the following.

Regarding a polycrystalline material as carbonyl iron or nickel with a uniform distribution of the anisotropic crystals inside the particle, an isotropic behavior is observed at the microscopic level where the particles are represented as a continuum. Thus, $\Psi^{\star}$ mag only depends on the norm of the Lagrangian magnetic field $|\boldsymbol{H}|$. In order to capture the characteristic saturation effect of ferromagnetic materials, the Langevin function

$$
\Psi^{* \operatorname{mag}}(\boldsymbol{H}):=-\frac{\mu_{0}}{2 \varrho_{0}} \int_{0}^{|\boldsymbol{H}|} M^{\mathrm{s}}\left[\operatorname{coth}(\alpha H)-(\alpha H)^{-1}\right] \mathrm{d} H,
$$

is used [30, 44]. Therein, $M^{\mathrm{s}}$ and $\alpha$ denote the saturation magnetization and a scaling 
factor, respectively. Experimental data for carbonyl iron and the fitted model with $M^{\mathrm{s}}=841 \mathrm{kA} \mathrm{m}^{-1}$ and $\alpha=2.18 \times 10^{-5} \mathrm{~mA}^{-1}$ are given in Figure 24.1(a).

\subsubsection{Magnetically hard particles}

In contrast to magnetically soft materials, the behavior of magnetically hard materials as NdFeB strongly depends on the load history. Thus, internal variables $\boldsymbol{Q}^{\alpha}$ are used to describe the actual state of the material.

A phenomenological constitutive model which is based on the work of Bergqvist [60] and has been published by Kalina et al. [13] is shown in the following. Again, polycrystalline materials with isotropic properties are regarded. Within the model, the pinning of domain wall motion is considered as the cause of hystereses in ferromagnets [61]. Since it is not sufficient to represent the pinning within a real polycrystalline material by only one pinning strength $k$, it is modeled by $N$ independent so called pseudoparticles [60]. Each of them is characterized by the individual strength $k^{\alpha}$ and a related weight $\omega^{\alpha}$ which can be interpreted as the probability that a magnetic moment in the material belongs to the respective pseudoparticle. In this case, the free energy function is represented by

$$
\varrho_{0} \Psi^{\mathrm{mag}}\left(\boldsymbol{B}, \boldsymbol{M}^{\alpha}\right):=-\sum_{\alpha=1}^{N} \omega^{\alpha} \boldsymbol{B} \cdot \boldsymbol{M}^{\alpha}+\sum_{\alpha=1}^{N} \omega^{\alpha} \int_{0}^{\left|\boldsymbol{M}^{\alpha}\right|} 1 / \gamma \tanh ^{-1}\left(M^{\alpha} / M^{\mathrm{s}}\right) \mathrm{d} M^{\alpha} .
$$

In the equation above, $\boldsymbol{M}^{\alpha}$ denotes a part of the magnetization belonging to the weight $\omega^{\alpha}$. According to the general constitutive relations given in Eq. (24.11), the equations

$$
\begin{aligned}
& \boldsymbol{H}=\frac{\partial \Omega}{\partial \boldsymbol{B}}=\frac{1}{\mu_{0} J} \boldsymbol{C} \cdot \boldsymbol{B}-\sum_{\alpha=1}^{N} \omega^{\alpha} \boldsymbol{M}^{\alpha} \text { and } \\
& \mathcal{D}=-\sum_{\alpha=1}^{N} \frac{\partial \Omega}{\partial \boldsymbol{M}^{\alpha}} \cdot \dot{\boldsymbol{M}}^{\alpha}=\sum_{\alpha=1}^{N} \omega^{\alpha} \boldsymbol{B}^{\mathrm{i} \alpha} \cdot \dot{\boldsymbol{M}}^{\alpha} \geq 0
\end{aligned}
$$

follow in which $\boldsymbol{B}^{\mathrm{i} \alpha}=\boldsymbol{B}-\gamma^{-1} \tanh ^{-1}\left(\left|\boldsymbol{M}^{\alpha}\right| / M^{\mathrm{s}}\right)\left|\boldsymbol{M}^{\alpha}\right|^{-1} \boldsymbol{M}^{\alpha}$ is defined as the irreversible induction field and $\mathcal{D}$ denotes the rate of dissipation. Applying the principle of maximum dissipation [62], the rate independent behavior of the material is given by the evolution equations for $\boldsymbol{M}^{\alpha}$, the switching conditions $\phi^{\alpha}$ and the related Karush-KuhnTucker conditions:

$$
\dot{\boldsymbol{M}}^{\alpha}=\lambda^{\alpha} \frac{\boldsymbol{B}^{\mathrm{i} \alpha}}{\left|\boldsymbol{B}^{\mathrm{i} \alpha}\right|} \quad, \phi^{\alpha}:=\left|\boldsymbol{B}^{\mathrm{i} \alpha}\right|-k^{\alpha} \quad \text { and } \quad \lambda^{\alpha} \phi^{\alpha}=0 \wedge \lambda^{\alpha} \geq 0 \wedge \phi^{\alpha} \leq 0 .
$$

Therein, $\lambda^{\alpha}$ and $k^{\alpha}$ denote a Lagrange parameter and the pinning strength, respectively. Experimental data for $\mathrm{NdFeB}$ and the adopted model with $N=10$ are shown in Figure 24.1(c). The fitted set of parameters $\omega^{\alpha}, k^{\alpha}, \gamma$ and $M^{\mathrm{s}}$ are given in Kalina et al. [13].

In order to incorporate the introduced constitutive model into a FE procedure according to subsection 24.2.2, the set of equations given in (24.29) has to be solved and 
the algorithmically consistent material tangent $\partial_{\Delta B} \Delta \boldsymbol{H}$ has to be calculated at each integration point. This is done by an implicit Euler scheme which is adapted from the theory of plasticity.

\subsubsection{Polymer matrix}

Besides the particles, the MAEs' effective behavior essentially depends on the properties of the polymer matrix. In order to achieve large coupling effects in the magnetoactive composite material, the stiffness of the matrix has to be preferably low but suitable regarding technical applications. A common choice for the production of MAEs are e.g. silicone elastomers [9-11, 47]. In order to consider a realistic behavior of such a material within the presented modeling approach, uniaxial tension tests for Zhermack silicone ZA 8 LT shore 8 from Troll Factory have been carried out. The geometry of the sample with circular cross section and the jig for the load application are taken from Dohmen [63].

\subsubsection{Sample production and testing}

The samples were manufactured by mixing a base liquid, a catalyst liquid, and varying amounts of silicone oil together. Furthermore, a slight amount of silicone-based white dye is added to colorize the samples for local strain measurements. To study the influence of silicone oil on the mechanical properties, five sets a six samples, with the oil volume fractions $\phi_{\text {oil }}=\{0,10,20,30,40\} \%$ were produced. The remaining volume fractions are distributed equally between base and catalyst. After assembling and stirring all chemicals into a beaker at room temperature, the compound was depressurized in a vacuum chamber for $10 \mathrm{~min}$. In each manufacturing cycle, the liquid suspension was filled into two separate polytetrafluoroethylene (PTFE)-moulds, where metal caps with holes-the connectors for later mechanical testing-are placed inside. After waiting at least $2 \mathrm{~h}$, the two samples are demoulded.

All manufactured samples were tested with a tensile test machine, where the local strains are measured via digital image correlation with the tool Aramis from GOM. To this end, patterns are sprayed on the sample surfaces, cf. the photograph in Figure 24.2(b). The maximum applied local stretch in the loading direction was $\lambda_{\|} \approx 2$. Before the recording of the stress-strain curves, all samples were preconditioned to avoid non-reproducibility due to the Mullins effect [64]. The samples were pulled with a displacement rate of $20 \mathrm{~mm} \mathrm{~min}^{-1}$. An increase of the rate up to $200 \mathrm{~mm} \mathrm{~min}^{-1}$ had almost no influence on the measured stress-strain curves. Thus, the material could be regarded as ideally elastic. Altogether, the measured stress-strain curves shown in Figure 24.2(a) reveal a highly nonlinear response for all amounts of silicone oil. Furthermore, the recorded perpendicular stretches $\lambda_{\perp}$ indicate a nearly ideal incompressible behavior within the examined strain range, cf. Figure 24.2(b). 
(a)

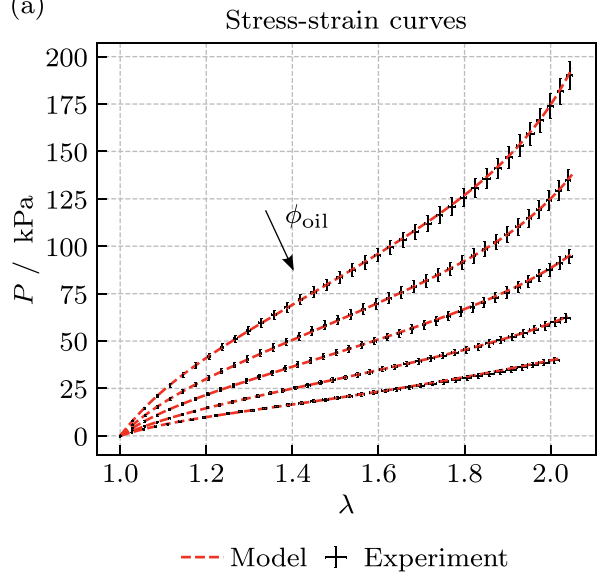

(b)

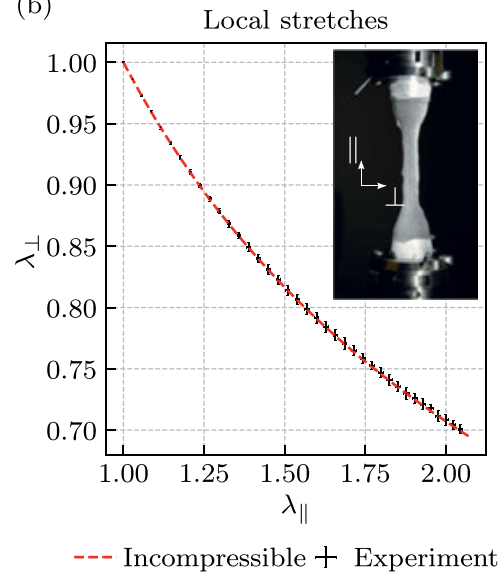

Figure 24.2: Characterization of a silicone elastomer by uniaxial tension tests: (a) experimentally determined stress strain curves for the amounts of silicone oil $\phi_{\text {oil }}=\{0,10,20,30,40\} \%$ and adjusted Ogden model (24.31), and (b) measured local stretches $\lambda_{\|}$and $\lambda_{\perp}$ for $\phi_{\text {oil }}=0 \%$ in the load direction and perpendicular as well as ideal incompressible stretch curve. The directions $\|$ and $\perp$ are shown in the photograph of a tested specimen with sprayed patterns for the local strain measurement.

Finally it turns out, that the resulting shear modulus of the polymer, given in Table 24.1, could be adjusted in a specific way by the amount of added silicone oil within the production process. The dependence of $\mu_{\text {init }}$ on $\phi_{\text {oil }}$ is however nonlinear and could be described by

$$
\mu_{\text {init }}\left(\phi_{\text {oil }}\right)=a \exp \left(-b \phi_{\text {oil }}\right)+c .
$$

The parameters in Eq. (24.30) are determined in such a way, that $\mu_{\text {init }}(100 \%)=0 \mathrm{kPa}$ and yield $a=103.91 \mathrm{kPa}, b=0.032$ and $c=-4.23 \mathrm{kPa}$.

Table 24.1: Identified parameters of the Ogden model (24.31) for different amounts of silicone oil $\phi_{\text {oil }}$ and related initial shear moduli $\mu_{\text {init }}$. The compression moduli $\kappa$ are given for a Poisson's ratio of $v=0.49$.

\begin{tabular}{lrrrrrrrr}
\hline $\boldsymbol{\phi}_{\text {oil }}$ & $\boldsymbol{\mu}_{\text {init }} / \mathbf{k P a}$ & $\boldsymbol{\mu}_{\mathbf{1}} / \mathbf{k P a}$ & $\boldsymbol{\mu}_{\mathbf{2}} / \mathbf{k P a}$ & $\boldsymbol{\mu}_{\mathbf{3}} / \mathbf{k P a}$ & $\boldsymbol{\alpha}_{\mathbf{1}}$ & $\boldsymbol{\alpha}_{\mathbf{2}}$ & $\boldsymbol{\alpha}_{\mathbf{3}}$ & $\boldsymbol{\kappa} / \mathbf{k P a}$ \\
\hline $0 \%$ & 99.17 & -22.67 & 24.17 & $9 \times 10^{-5}$ & -6.61 & 2 & 18.78 & $4.93 \times 10^{3}$ \\
$10 \%$ & 71.79 & -15.88 & 19.33 & $8.16 \times 10^{-5}$ & -6.58 & 2.03 & 18.29 & $3.56 \times 10^{3}$ \\
$20 \%$ & 52.09 & -11.78 & 12.31 & $4.07 \times 10^{-5}$ & -6.67 & 2.08 & 18.18 & $2.58 \times 10^{3}$ \\
$30 \%$ & 33.55 & -13.63 & 2.81 & - & -3.99 & 4.53 & - & $1.67 \times 10^{3}$ \\
$40 \%$ & 25.19 & -6.85 & 1.72 & - & -6.85 & 2.00 & - & $1.25 \times 10^{3}$ \\
\hline
\end{tabular}




\subsubsection{Model and parameter identification}

Within the microscopic modeling approach, the polymer matrix is regarded as nonmagnetizable. Furthermore, due to the negligible rate dependency of the characterized silicone elastomer, possible internal variables $\boldsymbol{Z}^{\alpha}$ do not have to be considered, so that $\Psi$ within $\mathcal{B}_{0}^{\mathrm{m}}$ only depends on $\boldsymbol{C}$. If the polymer is regarded as isotropic, it follows $\Psi(\boldsymbol{C})=\Psi\left(I_{1}, I_{2}, I_{3}\right)=\Psi\left(\lambda_{1}, \lambda_{2}, \lambda_{3}\right)$, where $I_{i}$ and $\lambda_{i}$ denote the invariants of the deformation tensor $\boldsymbol{C}$ and the principal stretches, respectively.

To describe the observed strongly nonlinear response, the ideal incompressible model

$$
\begin{aligned}
\varrho_{0} \Psi & =\varrho_{0} \Psi^{\mathrm{dev}}\left(\lambda_{1}^{\mathrm{iso}}, \lambda_{2}^{\text {iso }}, \lambda_{3}^{\text {iso }}\right)-p(J-1) \\
& :=\sum_{p=1}^{N} \frac{\mu_{p}}{\alpha_{p}}\left(\lambda_{1}^{\text {iso } \alpha_{p}}+\lambda_{2}^{\text {iso } \alpha_{p}}+\lambda_{3}^{\text {iso } \alpha_{p}}-3\right)-p(J-1)
\end{aligned}
$$

of the Ogden type is used, where $\lambda_{i}^{\text {iso }}:=\lambda_{i} J^{-1 / 3}$ are the isochoric principal stretches according to the Flory-split [65]. In the equation above, $\mu_{p}, \alpha_{p}$ and $p$ denote parameters of the Ogden model and the hydrostatic pressure, respectively. The adjusted model (31) and the measured uniaxial tension tests for all analyzed amounts of silicone oil are shown in Figure 24.2(a). Within the parameter identification, a model with $N=3$ sets has been chosen for $\phi_{\text {oil }}=\{0,10,20\} \%$. In case of $\phi_{\text {oil }}=\{30,40\} \%$, the adjustment is already accurate with $N=2$ sets. Furthermore, to describe the initial shear modulus $\mu_{\text {init }}$ exactly and to ensure a physically meaningful behavior, the conditions

$$
0=\frac{1}{2} \sum_{p=1}^{N} \alpha_{p} \mu_{p}-\mu_{\text {init }} \text { and } \alpha_{p} \mu_{(p)} \geq 0 \forall p \in 1 \ldots N
$$

are used in the least square fitting algorithm as constraints. The determined parameter sets are given in Table 24.1. Altogether, the model is able to describe the highly nonlinear behavior up to the measured strains of approximately $100 \%$ quiet well.

To incorporate the parameterized model into a standard FE simulation, according to section 24.2.2, a compressible model is used instead of the ideal incompressible one. Thus, the latter term in Eq. (24.31) is replaced by the volumetric energy function $\varrho_{0} \Psi^{\mathrm{vol}}(J):=\frac{\kappa}{4}\left(J^{2}-2 \ln J-1\right)$, with $\kappa$ denoting the initial compression modulus. In order to ensure a stable FE calculation with a nearly incompressible stress response, a Poisson's ratio of $v=0.49$ and, consequently, a compression modulus of $\kappa=2 / 3 \mu_{\text {init }}(1+v) /(1-2 v)$ is chosen. With that, the deviation to the parameterized incompressible model (24.31) is below $1.5 \%$ for stretches up to $\lambda=1.7$ and below $4.5 \%$ for the largest measured strains, respectively.

If only processes within the range of small or moderate strains are regarded, the stress-strain curve could be described by a comparatively simple neo-Hookean model which results from (24.31) for $N=1$ and $\alpha_{p}=2$. The corresponding Young's modulus follows to $E=2 \mu_{\text {init }}(1+v)$. 


\subsection{Study on the effective behavior of MAEs}

Besides the previously characterized properties of the particles and matrix, the effective behavior of MAEs results from the arrangement and the interplay of the components on the microscale. In order to analyze the microscopically-driven behavior, the homogenization scheme given in subsection 24.2.1.2 is used to discuss basic macroscopic effects in the following. This is done within three studies for MAEs filled with magnetically soft and hard particles, where the polymer matrix is described by a simplified neo-Hookean potential in a first step.

\subsubsection{Homogenization of magnetically soft MAEs}

At first, the resulting properties of magnetically soft MAEs are discussed. Thereby, the magnetic behavior of carbonyl iron is regarded and the polymer matrix is described by a reduced neo-Hookean model. Thus, the resulting effective behavior is fully reversible but highly nonlinear.

\subsubsection{Analyses of the MR effect}

One of the most popular properties of MAEs is their ability to change the effective stiffness if an external field is applied. This phenomenon, also known as the magnetorheological (MR) effect, is examined for 3D cubic and random microstructures with varying particle volume fractions within this subsection. The parameters of the neoHookean elastic model describing the polymer matrix are chosen to $E=100 \mathrm{kPa}$ and $v=0.49$.

In order to determine the magnetically induced MR-effect, several simulations have to be performed for each analyzed microstructure. If the behavior under shearing is investigated, this can be done by increasing an effective field $\overline{\boldsymbol{H}}$ to a specific value $\bar{H}^{\text {max }}$ in a first step and applying a shear deformation while holding $\overline{\boldsymbol{H}}$ constant, afterwards. Finally, the resulting effective mechanical shear stress which is related to the initial shear modulus $\bar{G}$ is calculated from the numerical homogenization. In order to ensure a virtual linear stress-strain-curve, a maximum shear deformation of $\bar{\gamma}=1 \%$ is applied. The described procedure is repeated for maximum magnetic fields $\bar{H}^{\text {max }}$ in the range of $0-2000 \mathrm{kA} \mathrm{m}^{-1}$, so that $\bar{G}$ could be determined as a function of $\overline{\boldsymbol{H}}$. According to the sketch given in Figure 24.3(b), the prescribed effective magnetic field and deformation are chosen as

$$
\left[\bar{H}_{K}\right]=\left[\begin{array}{c}
0 \\
\bar{H}^{\max } \\
0
\end{array}\right], \quad\left[\bar{F}_{l K}\right]=\left[\begin{array}{lll}
1 & \bar{\gamma} & 0 \\
0 & 1 & 0 \\
0 & 0 & 1
\end{array}\right],
$$


(a)

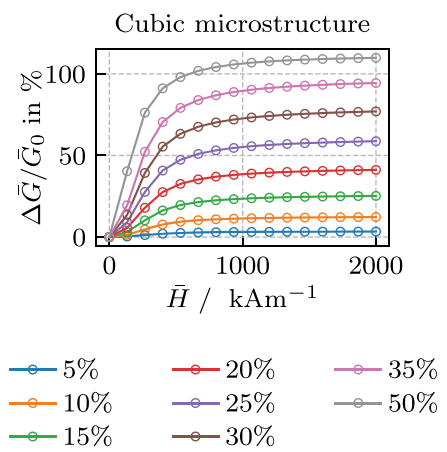

(b)
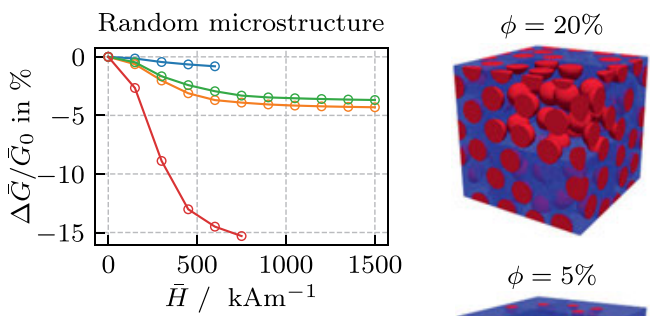

$\phi=5 \%$

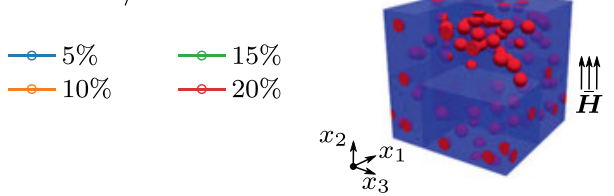

Figure 24.3: Predicted magnetorheological effect considering a simple shear deformation with $\bar{\gamma}=1 \%$ : (a) simplified cubic microstructures with different particle volume fractions $\phi$, and (b) random microstructure and considered 3D microstructures with 100 spherical particles.

where $[(\bullet)]$ denotes the specification of tensor coordinates with respect to a Cartesian frame. Consequently, the shear stress $\bar{P}_{21}$ is proportional to $\bar{G}$ which is changed by the magnetic loading. The FE meshes of the investigated cubic and random RVEs were generated with an in-house script for the tool HyperMesh. In case of the random RVE geometries with 100 included spherical particles, the minimum particle distance was set to $d / 5$ within the positioning algorithm, cf. Figure 24.3(b).

\section{Cubic microstructure}

Initially, the behavior of cubic microstructures with varying particle volume fractions $\phi=\{10,15,20,25,30,35,50\} \%$ are analyzed. For all volume fractions, a magnetically induced stiffening could be observed, where the magnitude of the MR-effect increases with the particle volume fraction, see Figure 24.3(a). Regarding the curve $\Delta \bar{G} / \bar{G}_{0}$ over $\bar{H}$, the typical quadratic behavior could be observed for small magnetic fields. For large values of $\bar{H}$, a clear saturation effect is visible which leads to an overall S-shaped curve for each particle volume fraction. The predicted curves are in qualitative accordance with experimental results $[66,67]$.

\section{Random microstructure}

Furthermore, the behavior of more realistic MAEs with underlying random particle distributions is investigated. Within the analyses, the particle volume fractions $\phi=\{5,10,15,20\} \%$ are investigated. Regarding the results given in Figure 24.3(b), several findings could be made. Equal to the cubic microstructure, the typical S-shaped curve is predicted. However, it stands out that the predicted MR-effect is negative for all considered microstructures which is in contradiction to experimental observations. 
Furthermore, the predicted effect seems not to increase in a systematic way, since e.g. the decrease in stiffness of $\phi=15 \%$ is less compared to the one of $\phi=10 \%$ whereas it again increases for $\phi=20 \%$. Results which demonstrate a similar unsystematic response of the composites' MR-effect are shown by Gebhart et al. [45] for 2D random microstructures.

Since the overall MR-effect depends on the microscopic magnetic interactionswhich increases for closer particle distances-and the properties of the polymer, a reason for the observed decrease in stiffness cannot be stated easily. Probably, a positive effect only occurs if the initial particle distance is reduced to lower values, because the magnetic attraction force increases nonlinear with respect to the particle distance $[24,68]$. However, this supposition cannot be confirmed at the moment, since the performed simulations are getting already unstable for the analyzed RVEs with a minimum particle distance of $d / 5$. Due to that, the simulated curves for $\phi=\{5,20\} \%$ are not completed up to $\bar{H}=1500 \mathrm{kAm}^{-1}$.

\subsubsection{Comparison of 2D and 3D simulations}

Due to the enormous computational cost of realistic 3D simulations which explicitly resolve the magnetizable particles, many computational analyses based on microscopic approaches are restricted to the 2D case. Therefore, in order to assess whether those predictions are still sensible and can provide an understanding of principle microscopic mechanisms inside MAEs, a comparison of 2D and 3D simulations according to Metsch et al. [30] is shown here. A simplified cubic microstructure containing only one spherical or even circular particle is investigated, whereby RVEs with the same geometry ( $\mathrm{Cubic}^{2 \mathrm{D}}$ and $\mathrm{Cubic}_{d}^{3 \mathrm{D}}$ ) as well as the same particle-volume fraction $\left(\mathrm{Cubic}^{2 \mathrm{D}}\right.$ and $\mathrm{Cubic}_{\phi}^{3 \mathrm{D}}$ ) of $\phi \approx 20 \%$ are compared.
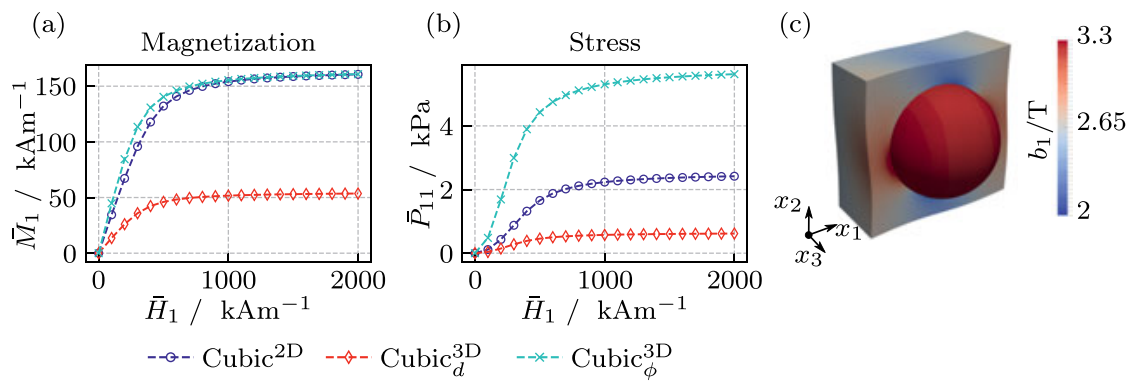

Figure 24.4: Comparison of cubic RVEs: (a) computed effective magnetization $\overline{\boldsymbol{M}}$ and (b) mechanical stress $\overline{\boldsymbol{P}}^{\text {tot }}$ for $\overline{\boldsymbol{F}}=\boldsymbol{I}$, and (c) local induction field within the 3D geometry Cubic ${ }_{\phi}^{3 \mathrm{D}}$ for shear loading. 
While the latter two RVEs predict a virtually coinciding $\bar{M}-\bar{H}$ curve, its maximum value is reduced by a factor of three for the $\mathrm{Cubic}_{d}^{3 \mathrm{D}}$ microstructure, cf. Figure 24.4(a). This effect is directly related to the differences in $\phi$, whereas the variations in Cubic ${ }^{2 D}$ and $\mathrm{Cubic}_{\phi}^{3 \mathrm{D}}$ are a result of differences in the related cylindrical and spherical inclusions.

Comparing the effective mechanical stresses for $\overline{\boldsymbol{F}}=\boldsymbol{I}$ in Figure 24.4(b), a qualitative agreement can be found for all RVEs, i.e. a positive, S-shaped component $\bar{P}_{11}$ in dependence of $\bar{H}_{1}$. In contrast to that, considerable differences become apparent in the quantitative results. Due to the differing demagnetization factors and the mechanical behavior of cylinders and spheres, the magnetically induced particle interactions vary considerably even if the particle volume fraction is held constant.

\subsubsection{Homogenization of magnetically hard MAEs}

In contrast to magnetically soft MAEs, the usage of magnetically hard fillers as NdFeB in the production process leads to composites with irreversible and strongly pathdependent behavior. For instance, measurements presented by Linke et al. [14] show, that the overall magnetization loops of MAEs based on a soft silicone elastomer matrix filled with $\mathrm{NdFeB}$ are significantly smaller compared to the loops of NdFeB particles fixed in a stiff epoxy resin matrix, cf. Figure 24.5(a). In the following, the results presented in Kalina et al. [13,69] are summarized. Therein, the elastomer matrix is represented by a neo-Hookean model with $E=100 \mathrm{kPa}$ and $v=0.49$.

(a)

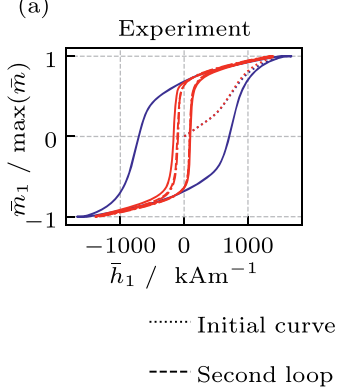

(b)

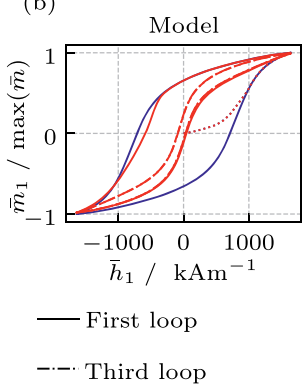

(c)

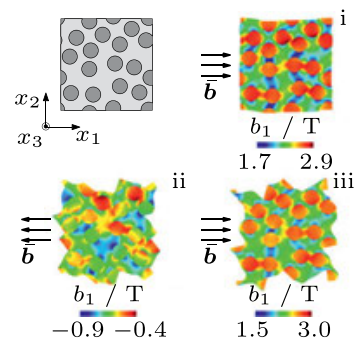

Figure 24.5: Magnetic hystereses of composites filled with NdFeB particles: (a) measured effective behavior of epoxy sample (blue lines) and MAE sample (red lines) with a low modulus elastomer of $E^{\mathrm{m}} \approx 64 \mathrm{kPa}$, (b) calculated hysteresis loops for epoxy and MAE with $E^{\mathrm{m}}=100 \mathrm{kPa}$, and (c) evolution of the MAE microstructure during the first hysteresis loop and local $b_{1}$-field. Experimental data are taken from Linke et al. [14] and Kalina et al. [13]. 


\subsubsection{Magnetization loops}

In order to investigate the effective behavior, numerical homogenizations with RVEs containing randomly distributed circular inclusions are examined. During the simulation, the macroscopic deformation is fixed, i.e. $\overline{\boldsymbol{F}}=\boldsymbol{I}$. The predicted initial magnetization curve as well as the first and second hysteresis loops of the MAE are compared to the hysetereses of a composite with $\mathrm{NdFeB}$ particles embedded into an epoxy resin matrix, see Figure 24.5(b). In accordance with the measurement, the second major hysteresis loop of the MAE is significantly smaller.

As demonstrated in Kalina et al. [13] from a theoretical point of view, this effect results from magnetically induced particle rotations within the polymer. Due to these rotations, the inversion of the MAE's overall magnetization is expedited compared to the epoxy sample, in which the inversion completely results from local domain switching processes within the mechanically fixed particles. Furthermore, the particle rotations insert stored strain energy into the polymer matrix. This energy storage appears after the first inversion of the external magnetic field and leads to an inverse rotation of the particles if the external field is equal to zero. Thus, the switching of $\overline{\boldsymbol{m}}$ within the following ascending branch and the second hysteresis loop is faster than the initial process and consequently, an asymmetrically shaped first hysteresis loop occurs. Finally, the simulations indicate that the reversion of $\overline{\boldsymbol{m}}$ is a result of particle rotations and internal domain conversion processes. The evolution of the MAE microstructure during the first hysteresis loop and the related local $b_{1}$-field are given in Figure 24.5(c).
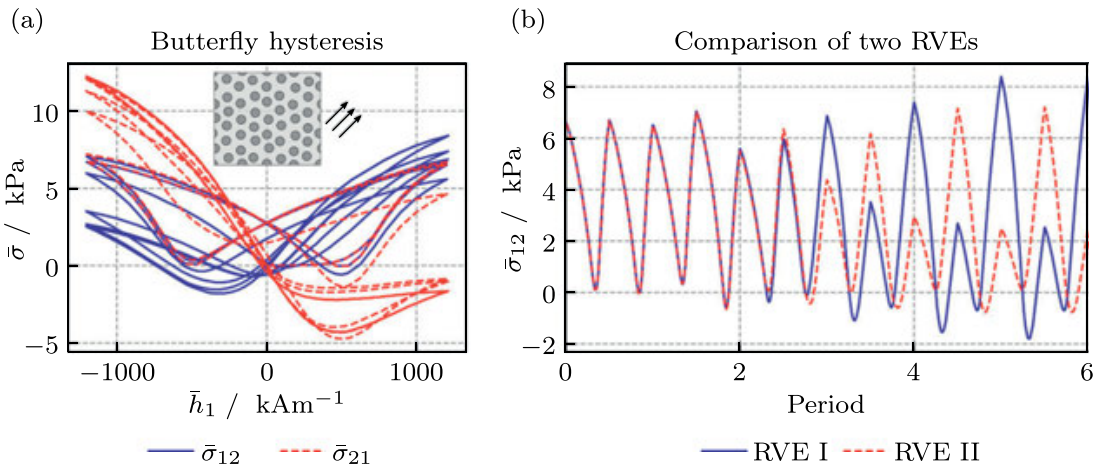

Figure 24.6: Simulation of a magnetically hard MAEs: (a) mechanical butterfly hystereses for an alternating magnetic load in the $45^{\circ}$-direction, and (b) comparison of the stress response of two RVEs with almost identical microstructure which only differs in the initial position of one single particle. 


\subsubsection{Mechanical hysteresis loops}

To analyze the mechanical response of magnetically hard MAEs, imperfect hexagonal microstructures, i.e. perfectly hexagonal distributions modified by a small geometric perturbation of the particles reference position, cf. the microstructure shown in Figure 24.6(a). They are loaded with an alternating effective induction $\overline{\boldsymbol{B}}$ in the $45^{\circ}$ direction where the effective deformation $\overline{\boldsymbol{F}}$ is fixed.

Regarding the resulting mechanical stress response of the system, butterfly hystereses appear. The loops reveal a stress $\overline{\boldsymbol{\sigma}}$ which is initially symmetric and gets asymmetric after several load cycles, see $\bar{\sigma}_{12}$ and $\bar{\sigma}_{21}$ in Figure 24.6(a). As in the case of magnetic hysteresis, this is an effect of the local particle rotations in the mathematically positive or negative direction, respectively. Since these rotations are not in equilibrium, a resulting magnetically-induced torque, which has to be compensated by a non-symmetric mechanical stress tensor is observed. Furthermore, the effect of smaller hystereses, appearing in the system's magnetic response, also appears in the butterfly loops. The described effects become dominant with an increase of the local particle rotations. However, after five load cycles a constant hysteresis is reached in the system's following loading periods.

Finally, the magneto-mechanical response is calculated for two nearly identical imperfect hexagonal RVEs which differ in the reference position of only one particle. While the magnetic answer of both systems almost coincides and is thus not depicted, a strong variation of the mechanical response is detected when the microstructural particle rotations become dominant, cf. Figure 24.6(b). According to that, the presented study indicates a non-systematic behavior with respect to the stress response.

\subsection{Macroscopic modeling approach}

In the previous section, it is shown that the effective behavior of MAEs can be calculated by computational homogenizations. However, in order to simulate the behavior of real MAE samples under complex loading conditions in a computationally efficient way, it is necessary to use a macroscopic approach.

\subsubsection{Macroscopic models}

In this subsection, a macroscopic model similar to Kalina et al. [44] is discussed and extended for the magnetically nonlinear case. In contrast to the most previously published approaches in this field, it is constructed and parameterized by using results from computational homogenizations. As a consequence, the model contains the properties of the microstructure implicitly and is nonetheless computationally efficient 
compared to costly $\mathrm{FE}^{2}$-schemes [34, 35]. The described strategy is often denoted as decoupled multiscale scheme [70].

\subsubsection{Microscopic simulations-Generation of the data basis}

The macroscopic modeling approach is realized for the description of $2 \mathrm{D}$ isotropic MAEs with an underlying random microstructure, where the minimum particle-particle distance is set to 1.1 times the diameter. For statistical purpose, 10 monodisperse RVEs, each containing $N=400$ particles are analyzed. Exemplarily, the volume fraction is chosen to $\phi=30 \%$ in the following. The reader is referred to Kalina et al. [44] for a study which evaluates several particle volume fractions.

A suitable database for the parameter identification of the macroscopic model is generated by three purely mechanical load cases (M1-M3) and six coupled magnetomechanical load cases (MM1-MM6), cf. the schematic depiction in Figure 24.7(b). Within
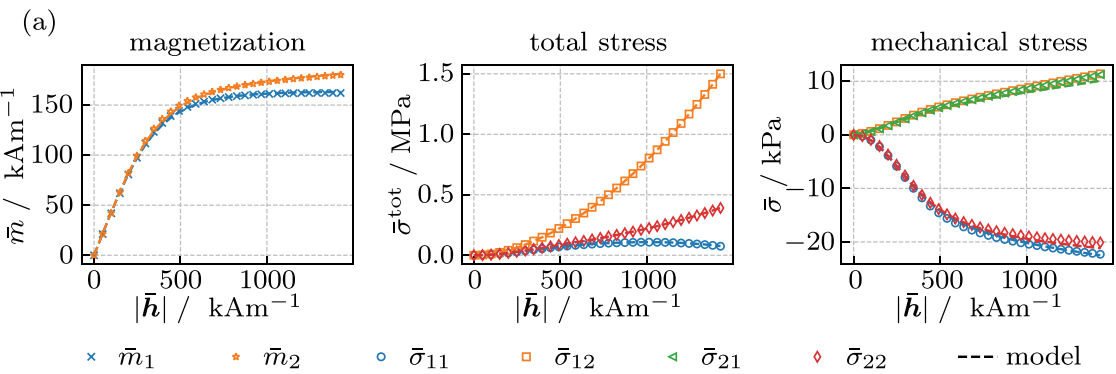

(b)

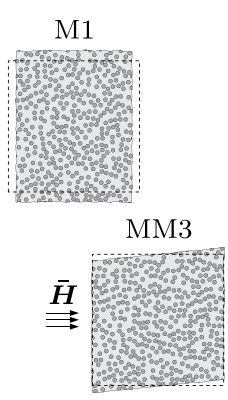

M2

M3

MM1 MM2
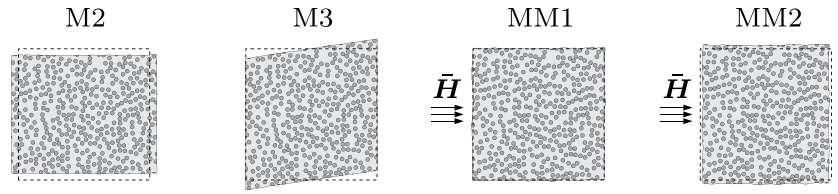

(c)
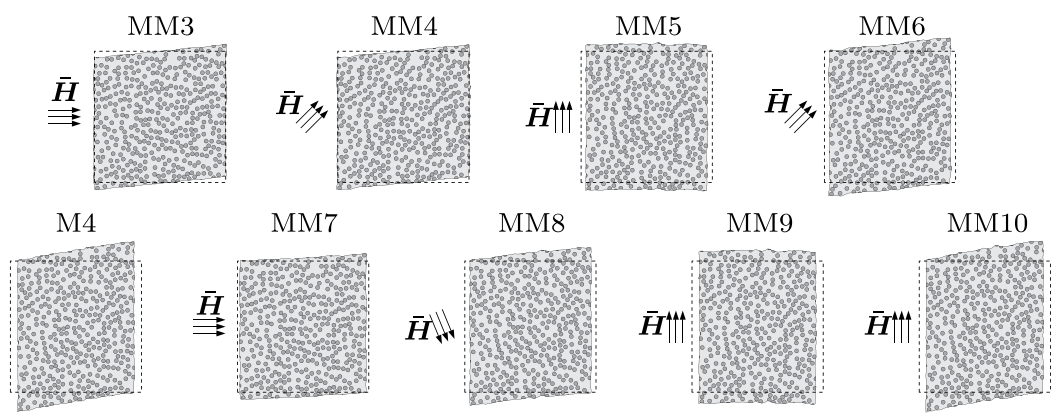

Figure 24.7: Multiscale modeling approach: (a) averaged homogenized stresses $\overline{\boldsymbol{\sigma}}^{\text {tot }}$ and $\overline{\boldsymbol{\sigma}}$ as well as magnetization $\overline{\boldsymbol{m}}$ of an RVE with $\phi=30 \%$ and adapted macroscopic model (24.36) plus (24.38, 24.39), (b) mechanical load cases M1-M3 and coupled magneto-mechanical load cases MM1-MM6 for calibration, as well as (c) load cases for validation. 
the coupled load cases, the effective magnetic field $\overline{\boldsymbol{H}}$ and deformation $\overline{\boldsymbol{F}}$ are applied simultaneously, where the maximum effective stretches and shears are prescribed to different values: $\bar{\lambda}^{\max }=\{1.05,1.1,1.15\}$ and $\bar{\gamma}^{\max }=\{0.05,0.1,0.15\}$. With that, an accurate description of the macroscopic model in a wide range of deformations is guaranteed. The maximum effective field is applied to $\left|\overline{\boldsymbol{H}}^{\mathrm{max}}\right|=1500 \mathrm{kAm}^{-1}$. Load cases for the validation of the model, M4 and MM7-MM10, are shown in Figure 24.7(c).

Within the microscopic simulations, the properties of the constituents are described by the behavior of magnetically soft carbonyl iron particles according to Figure 24.1(b) and a silicone elastomer with $\phi_{\text {oil }}=40 \%$ which is described by the Ogden model (24.31), see the stress-strain curve given in Figure 24.2(a).

\subsubsection{Model description}

In the macroscopic constitutive model, the general split of the amended free energy $\bar{\Omega}^{\star}(\overline{\boldsymbol{C}}, \overline{\boldsymbol{H}})$ according to Eq. (24.12) into material dependent part $\bar{\varrho}_{0} \bar{\Psi}^{\star}(\overline{\boldsymbol{C}}, \overline{\boldsymbol{H}})$ and free space part $\bar{\Omega}^{* \text { free }}(\overline{\boldsymbol{C}}, \overline{\boldsymbol{H}})=-\mu_{0} / 2 \overline{\boldsymbol{J C}}^{-1}:(\overline{\boldsymbol{H}} \otimes \overline{\boldsymbol{H}})$, that does not depend on any material properties, is applied. To capture the effects of the underlying microstructure which is not resolved within the macroscopic approach, magneto-mechanical coupling terms have to be incorporated in $\bar{\Psi}^{\star}(\overline{\boldsymbol{C}}, \overline{\boldsymbol{H}})$. Regarding an isotropic, purely elastic and magnetically soft MAE, it can be described in terms of the following six magnetomechanical invariants:

$$
\begin{aligned}
& \bar{I}_{1}^{\text {iso }}:=\bar{J}^{-2 / 3} \operatorname{tr} \overline{\boldsymbol{C}}, \bar{I}_{2}^{\text {iso }}:=\frac{\bar{J}^{-4 / 3}}{2}\left[\operatorname{tr}^{2} \overline{\boldsymbol{C}}-\operatorname{tr}\left(\overline{\boldsymbol{C}}^{2}\right)\right], \bar{I}_{3}:=\bar{J}^{2}, \\
& \bar{I}_{4}:=|\overline{\boldsymbol{H}}|^{2}, \bar{I}_{5}:=\overline{\boldsymbol{C}}^{-1}:(\overline{\boldsymbol{H} \otimes \boldsymbol{H}}), \bar{I}_{6}:=\overline{\boldsymbol{C}}^{-2}:(\overline{\boldsymbol{H}} \otimes \overline{\boldsymbol{H}}) .
\end{aligned}
$$

To facilitate a systematic discussion of the macroscopic constitutive model, the specific Helmholtz free energy function is decomposed into purely mechanic, coupling and purely magnetic parts:

$$
\bar{\Psi}^{*}(\overline{\boldsymbol{C}}, \overline{\boldsymbol{H}}):=\Psi^{* \text { mech }}\left(\bar{I}_{1}^{\text {iso }}, \bar{I}_{2}^{\text {iso }}, \bar{I}_{3}\right)+\Psi^{* \operatorname{coup}}\left(\bar{I}_{1}^{\text {iso }}, \bar{I}_{2}^{\text {iso }}, \ldots, \bar{I}_{6}\right)+\Psi^{* \operatorname{mag}}\left(\bar{I}_{4}\right) .
$$

The chosen ansatzes are discussed in the following, where a magnetically linear model according to [44] and a magnetically nonlinear one are presented.

In the absence of magnetic fields, the MAE's behavior is characterized by the purely mechanical part of the model. To capture the nonlinear stress-strain behavior for effective stretches in the range of $\bar{\lambda}=0.9$ up to 1.15 . This part is described by an Ogden type model, so that the mechanical invariants have to be replaced by the principal stretches $\bar{\lambda}_{i}^{\text {iso }}$ and $\bar{J}$. With that, the mechanical part is given by 


$$
\bar{\varrho}_{0} \bar{\Psi}^{* \text { mech }}:=\sum_{p=1}^{N} \frac{\mu_{p}}{\alpha_{p}}\left(\sum_{i=1}^{3} \bar{\lambda}_{i}^{\text {iso }^{\alpha_{p}}}-3\right)+\frac{K}{4}\left(\bar{J}^{2}-\ln \bar{J}^{2}-1\right) .
$$

Note that the effective nonlinear stress-strain behavior results from the Ogden model, which is used within the microscopic simulations, cf. Figure 24.2. To reduce the number of parameters in (24.36), only $N=1$ term is used.

Using Eq. (24.34) and keeping the properties of the predicted macroscopic magneto-mechanical MAE response in mind, i.e. a quadratic dependence of $\bar{\Psi}^{\text {coup }}$ and $\bar{\Psi}^{\text {mag }}$ on the magnetic field for $|\overline{\boldsymbol{H}}| \rightarrow 0$, the linear model with

$$
\mu_{0}^{-1} \bar{\varrho}_{0} \bar{\Psi}^{* \text { coup }}:=-C_{1} \bar{I}_{5}+C_{2} \bar{I}_{6}+C_{3}(\bar{J}-1) \bar{I}_{6} \text { and } \bar{\varrho}_{0} \bar{\Psi}^{* \text { mag }}:=\mu_{0} C_{4} \bar{I}_{4}
$$

is chosen. It has shown to be suitable for the description of the deformation dependent effective behavior within the examined range of magneto-mechanical load cases but is only valid for small magnetic fields up to $|\overline{\boldsymbol{H}}| \approx 250 \mathrm{kAm}^{-1}$. A possible extension of the linear model (24.37) for large values of $|\overline{\boldsymbol{H}}|$ is given by the nonlinear model with

$$
\begin{aligned}
\mu_{0} \bar{\Psi}^{* \text { coup }:=} & -\frac{\gamma_{1}}{\delta_{1}} \ln \left[\cosh \left(\mu_{0} \delta_{1} \sqrt{\bar{I}_{5}}\right)\right]+\gamma_{2} \ln \left(1+\mu_{0}^{2} \delta_{2} \bar{I}_{6}\right) \ldots \\
& +\frac{1}{2} y_{3}(\bar{J}-1) \tanh ^{2}\left(\mu_{0} \delta_{3} \sqrt{\bar{I}_{5}}\right) \text { and } \\
\mu_{0} \bar{\Psi}^{* \text { mag }}:= & \gamma_{4} \ln \left(1+\mu_{0}^{2} \delta_{4} \bar{I}_{4}\right) .
\end{aligned}
$$

Therein, the well-known saturation behavior that results from the magnetization of the ferromagnetic particles, cf. Figure 24.1(b) is incorporated. The chosen functions enable the accurate description of the magnetization as well as the stress tensors, respectively. Altogether, a total number of 11 parameters have to be determined in the nonlinear case. In contrast to other macroscopic constitutive MAE models, e.g. Bustamante et al. [71] or Haldar et al. [41], a volumetric sensitivity has been added to $\bar{\Psi}^{\text {coup }}$.

\subsubsection{Parameter identification}

Besides the development of the macroscopic model, the crucial task in the presented approach is the identification of the mechanical, coupling and magnetic model parameter sets-in the following denoted as $\underline{\boldsymbol{\kappa}}^{\text {mech }}, \underline{\boldsymbol{\kappa}}^{\text {coup }}$ and $\underline{\boldsymbol{\kappa}}^{\text {mag }}$, respectively. A high accuracy of the models (24.36) plus (24.37) and $(24.36)$ plus $(24.38,24.39)$ is ensured if they describe the effective homogenized magnetization $\overline{\boldsymbol{m}}$ as well as the total and mechanical stress tensors $\overline{\boldsymbol{\sigma}}^{\text {tot }}$ and $\overline{\boldsymbol{\sigma}}$ in the linear and nonlinear magnetic regime, respectively.

The proposed parameter identification according to Kalina et al. [44] features a stepwise algorithm based on four least square optimizations. At first, the parameter 
sets $\underline{\boldsymbol{\kappa}}^{\text {mech }}, \underline{\boldsymbol{\kappa}}^{\text {coup }}$ and $\underline{\boldsymbol{\kappa}}^{\text {mag }}$ are identified independently from $i=1 \ldots N$ homogenized data sets defined by the correlated tensor fields $\left\{\overline{\boldsymbol{F}}_{i}, \overline{\boldsymbol{H}}_{i}\right\} \rightarrow\left\{\overline{\boldsymbol{b}}_{i}, \overline{\boldsymbol{\sigma}}_{i}{ }^{\text {tot }}\right\}$. Thus, the following three separate optimizations have to be performed:

(i.i) Determine $\underline{\boldsymbol{\kappa}}^{\text {mech }}$ from the purely mechanical load cases,

(i.ii) find $\underline{\boldsymbol{\kappa}}^{\text {coup }}$ from the magneto-mechanical load cases by setting $\underline{\boldsymbol{\kappa}}^{\text {mech }}$ from (i.i) constant, and

(i.iii) identify $\underline{\boldsymbol{\kappa}}^{\text {mag }}$ from the magneto-mechanical load cases by fixing $\underline{\boldsymbol{\kappa}}^{\text {coup }}$ from the step (i.ii).

With that, both, the total stress tensor $\overline{\boldsymbol{\sigma}}^{\text {tot }}$ and the magnetization $\overline{\boldsymbol{m}}$ are described with high accuracy. However, since $\mathcal{O}(\overline{\boldsymbol{\sigma}}) \approx 10^{-2} \mathcal{O}\left(\overline{\boldsymbol{\sigma}}^{\text {tot }}\right)$, see Figure 24.7(a), the mechanical stress tensor $\overline{\boldsymbol{\sigma}}=\overline{\boldsymbol{\sigma}}^{\text {tot }}-\overline{\boldsymbol{\sigma}}^{\mathrm{m}}$ is not captured with sufficient precision using the identified set of parameters $\underline{\boldsymbol{\kappa}}^{\text {coup }}$ and $\underline{\boldsymbol{\kappa}}^{\text {mag }}$. Therefore, the quantities $\overline{\boldsymbol{\sigma}}^{\text {act }}:=\overline{\boldsymbol{\sigma}}-\overline{\boldsymbol{\sigma}}^{\text {mech }}$ and $\overline{\boldsymbol{m}}$ are fitted simultaneously according to the final optimization

$$
\begin{aligned}
\left\{\underline{\boldsymbol{\kappa}}^{\text {coup }}, \underline{\boldsymbol{\kappa}}^{\mathrm{mag}}\right\}= & \arg \left\{\operatorname { m i n } _ { \underline { \boldsymbol { \kappa } } ^ { \text { coup } } \underline { \underline { \boldsymbol { m } } } ^ { \mathrm { mag } } } \sum _ { i = 1 } ^ { N } \left(W_{i}^{\sigma}\left\|\mid \overline{\boldsymbol{\sigma}}^{\mathrm{act}}\left(\overline{\boldsymbol{F}}_{i}, \overline{\boldsymbol{H}}_{i}, \underline{\boldsymbol{\kappa}}^{\mathrm{mech}}, \underline{\boldsymbol{\kappa}}^{\mathrm{coup}}, \underline{\boldsymbol{\kappa}}^{\mathrm{mag}}\right)-\overline{\boldsymbol{\sigma}}_{i}^{\mathrm{act}}\right\|^{2} \ldots\right.\right. \\
& \left.\left.+W_{i}^{m}\left|\overline{\boldsymbol{m}}\left(\overline{\boldsymbol{F}}_{i}, \overline{\boldsymbol{H}}_{i}, \underline{\boldsymbol{\kappa}}^{\text {coup }}, \underline{\boldsymbol{\kappa}}^{\mathrm{mag}}\right)-\overline{\boldsymbol{m}}_{i}\right|^{2}\right)\right\}
\end{aligned}
$$

which is denoted as step (ii) in the following. Therein, $W_{i}^{m}$ and $W_{i}^{\sigma}$ are weighting factors for the single error sums which are determined as the inverse of the maximum value of the components $\bar{\sigma}_{k l}^{\text {act }}$ and $\bar{m}_{k}$ for each load case, respectively. Step (ii) naturally causes an optimization of the model with respect to both fields, $\overline{\boldsymbol{\sigma}}$ and $\overline{\boldsymbol{m}}$, but also guarantees an accurate description of $\overline{\boldsymbol{\sigma}}^{\text {tot }}$. The determined values from the steps (i.i)-(i.iii) are used as starting values in the highly nonlinear optimization.

The procedure is exemplarily applied for the nonlinear model (36) plus (38, 39). In order to determine the parameter sets $\underline{\boldsymbol{\kappa}}^{\text {mech }}, \underline{\boldsymbol{\kappa}}^{\text {coup }}$ and $\underline{\boldsymbol{\kappa}}^{\text {mag }}$, the load cases M1-M3 as well as MM1-MM6 are used. With that, the parameters follow to the values given in Table 24.2. Figure 24.7(a) exemplarily depicts the calibrated model and the homogenized data for the load case MM4. The remaining load cases M4 and MM7-MM10 are used to validate

Table 24.2: Identified parameters of the macroscopic mechanical model (36) as well as the nonlinear magnetic model (38 and 39). The isotropic model is adapted to

\begin{tabular}{|c|c|c|c|c|c|c|c|}
\hline$K / \mathrm{MPa}$ & & $\mu_{1} / \mathrm{kPa}$ & $\alpha_{1} /-$ & & & & \\
\hline 1.80 & & -8.33 & -13.01 & & & & \\
\hline$\gamma_{1} / T$ & $\delta_{1} / \mathrm{T}^{-1}$ & $\gamma_{2} / \mathrm{T}^{2}$ & $\delta_{2} / T^{-2}$ & $\gamma_{3} / \mathrm{T}^{2}$ & $\delta_{3} / T^{-1}$ & $\zeta_{1} / T^{2}$ & $\xi_{1} / T^{-2}$ \\
\hline 33 & 2.65 & $7.32 \times 10^{-03}$ & 6.76 & $2.97 \times 10^{-02}$ & 2.40 & $1.30 \times 10^{-02}$ & 5.77 \\
\hline
\end{tabular}
homogenized data of an RVE with random particle distribution for $\phi=30 \%$. 
the fitted model which achieves errors below $15 \%$ for all considered load cases and field strengths. Altogether, the developed macroscopic approach is able to reproduce the homogenized response with a good accuracy up to the highly nonlinear magnetic regime.

\subsubsection{Simulation of the magnetostrictive effect}

With the microscopically based, adapted macro-model, the magnetostrictive (MS) effect of MAEs, i.e. the free deformation of a sample due to an external magnetic field, is analyzed. According to Figure 24.8(a), it is defined as

$$
\bar{\varepsilon}=\frac{l-l_{0}}{l_{0}}
$$

with $l$ and $l_{0}$ denoting the sample length in the reference and deformed configurations. Within the macroscopic simulation, the sample is embedded into a free space domain $\overline{\mathcal{F}}_{0}$ of adequate size, which enables the application of a homogeneous, external farfield $\overline{\boldsymbol{h}}^{\infty}$ [35, 44, 45, 72], see Figure 24.1(a). To this end, $\bar{\varphi}=\widehat{\bar{\varphi}}$ is prescribed on the exterior boundary $\partial \overline{\mathcal{F}}_{0}$ of the free space. Furthermore, displacements on $\partial \overline{\mathcal{F}}_{0}$ are prevented for simplicity, which however does not lead to a loss of generality [73]. Since the Lagrangian description of the BVP requires the calculation of a deformation field within the whole domain, $\overline{\mathcal{F}}$ is described as a neo-Hookean-elastic and nonmagnetizable medium with $\bar{E}^{\text {free }}=1 \mathrm{kPa}$ and $\bar{v}^{\text {free }}=0.4$ : This guarantees a negligible mechanical influence on the sample. In order to analyze the influence of the MAEs' geometry, elliptical samples with aspect ratios shifted from values between $l_{0} / h_{0}=1 / 4$ and 4 are considered, where $h_{0}$ is the sample's height. For the loading of the samples, a maximum far-field of $|\overline{\boldsymbol{h}}|^{\infty}=1500 \mathrm{kAm}^{-1}$ is applied in the horizontal direction.

Evaluating the FE simulations, a positive MS effect is predicted for all geometries, where, according to Figure 24.8(b), the maximum elongation appears for a circular sample

(a)

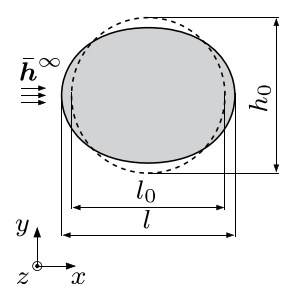

(b)

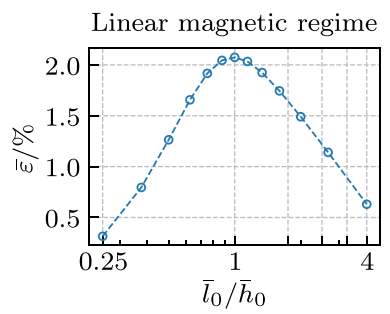

(c)

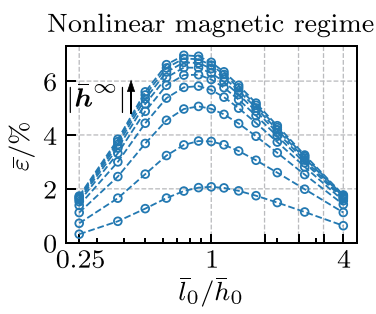

Figure 24.8: FE simulation of MS effects in elliptical MAE samples: (a) undeformed configuration (dashed lines) and deformed geometry (solid lines) for a circular sample, (b) and (c) overall MS strain $\bar{\varepsilon}=\frac{l-l_{0}}{l_{0}}$ depending on the sample shape. In (b), the linear magnetic regime for $\left|\bar{h}^{\infty}\right|=300 \mathrm{kAm}^{-1}$ and in (c), the nonlinear magnetic regime for $\left|\bar{h}^{\infty}\right|=\{300,450, \ldots 1500\} \mathrm{kAm}^{-1}$ are depicted, respectively. 
(a)

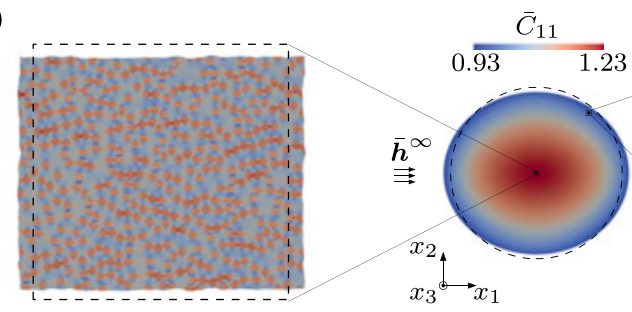

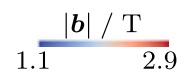

(b)

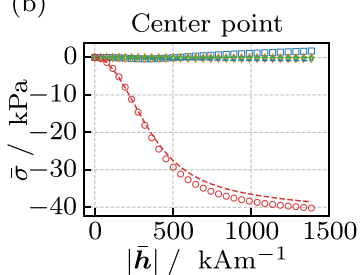

․ $\bar{\sigma}_{11}$

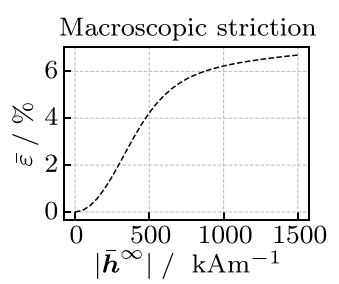

$\bar{\sigma}_{12}$

$\bar{\sigma}_{21}$

$\circ \bar{\sigma}_{22}$
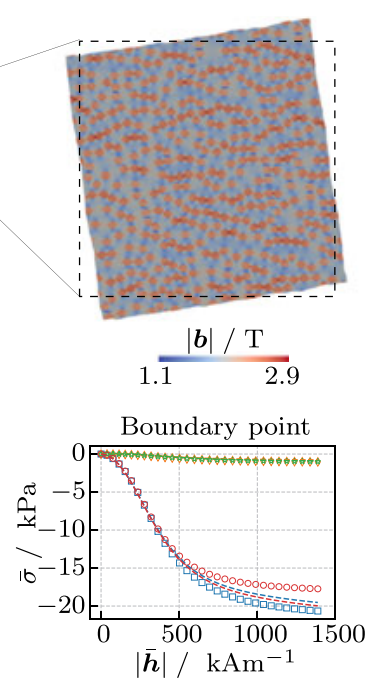

--- model

Figure 24.9: Decoupled multiscale scheme for a circular MAE: (a) inhomogeneous deformation of the sample due to an applied external field $\overline{\boldsymbol{h}}^{\infty}$ and local $\boldsymbol{b}$-field in the corresponding RVE at the center and a boundary point. In (b): comparison of the RVE's effective response to the macro-model according to Eqs. (24.36), (24.38) and (24.39) for localization BCs at these two points and macroscopic elongation $\bar{\varepsilon}=\Delta l / l_{0}$ of the sample.

within the linear magnetic regime at $\left|\overline{\boldsymbol{h}}^{\infty}\right|=300 \mathrm{kAm}^{-1}[44,45]$. Increasing the external field to values where saturation effects are relevant, the situation changes. Now, the maximum effect is predicted for the sample with a ratio $l_{0} / h_{0}=3 / 4$, see Figure 24.8 (c). This shift of the maximum MS effect from the ratio $1 / 1$ to a value below is in good agreement with results of Keip and Rammbausek [35], who perform $\mathrm{FE}^{2}$-simulations for periodic microstructures, at the saturation point. According to the presented findings, a significant correlation of the sample geometry and the resulting magneto-mechanical response is apparent. Consequently, the overall behavior results from microstructural as well as macroscopic properties and a separation of both effects is hardly achievable in experiments. Furthermore, strongly inhomogeneous deformations $\overline{\boldsymbol{F}}$ occur inside the sample even for elliptical or, in the 3D case, ellipsoidal geometries, cf. Figure 24.9(a).

Finally, in order to validate the macroscopic approach, the decoupled multiscale scheme is applied exemplarily for the circular sample. To this end, the effective deformation gradient $\overline{\boldsymbol{F}}$ and magnetic field $\overline{\boldsymbol{H}}$ in quadrature points close to the sample's center and a boundary point at an angle of $45^{\circ}$ starting from the $x_{1}$-axis are stored within the macroscopic FE simulation, respectively. The temporal evolutions of these tensor fields are applied in a computational homogenization to the RVE - this process is called localization. With that, the prediction of the macroscopic model can be compared to the 
response of the RVE. The norm of the local $\boldsymbol{b}$-field in the deformed RVEs and the calculated effective mechanical stress $\overline{\boldsymbol{\sigma}}$ of the macro-model and the homogenization are shown in Figure 24.9(a) and (b), respectively. Therein, only small deviations can be seen. This confirms the reliability of the macroscopic approach.

\subsection{Conclusions}

In this contribution, a continuum-based multiscale modeling approach for MAEs is presented. Applying the FE method for the solution of the nonlinear magneto-mechanical BVP, these materials are described on the micro- as well as the macroscale, where the two scales are connected via a computational homogenization scheme. The present work summarizes results given in Metsch et al. [29, 30] and Kalina et al. [13, 28, 44] and complements these findings at several points.

In order to incorporate realistic properties of the constituents on the microscale, experimental data are used to parameterize models for magnetically soft and hard filler particles as well as an elastic silicone elastomer matrix. For all of them, the chosen models are able to describe the measured response with high accuracy. By performing computational homogenizations, the effective behavior of magnetically soft as well as hard MAEs is predicted and qualitatively compared to experimental results. In the case of magnetically soft MAEs, the well-known magnetorheological effect is analyzed for 3D cubic and random microstructures. Furthermore, realistic 3D and simplified 2D simulations are compared, where a qualitative agreement but a significant quantitative deviation of both approaches is observed. In the case of magnetically hard MAEs, the experimentally determined effect of smaller hysteresis [14] is analyzed and can be explained by particle rotations on the microscale. In addition, a non-systematic behavior with respect to the MAEs' mechanical response is detected. Finally, a decoupled multiscale approach [70] which implicitly includes the microstructures' influence is presented for isotropic magnetically soft MAEs. This includes the summary of the magnetically liner model given in Kalina et al. [44] and an expansion to the magnetically nonlinear case, wherein saturation effects are considered. Exemplarily, the nonlinear model is calibrated by using homogenized data of $2 \mathrm{D}$ random microstructures for several load cases. The validity of the parameterized macroscopic model could be verified by further load cases. With the calibrated macroscopic model, the MS effect of several macroscopic samples are analyzed and compared with experiments.

Altogether, the presented modeling strategy has shown to be an adequate tool to predict the effective behavior of magnetically soft and hard MAEs, where the material is described without simplifications as the dipole assumption. Furthermore, the developed decoupled multiscale approach enables the implicit embedding of the microstructures' influence on the macroscale. A disadvantage of the strategy are however the necessary microscopic simulations for the calibration of the macroscopic model which are still quite costly, especially in the $3 \mathrm{D}$ case. The presented modeling strategy is 
adoptable for other particle-matrix-based magneto-active composites as e.g. ferrogels, see Birster et al. [74], Gebhart and Wallmersperger [72], Gebhart et al. [45] or Weeber et al. [75]. An essential task for the future is the expansion of the modeling approach to MAEs with chain-like microstructures. Furthermore, geometric data of real microstructures [16] should be used as input instead of artificially constructed distributions.

Acknowledgments: The computations were performed on a PC-Cluster at the Center for Information Services and High Performance Computing (ZIH) at TU Dresden. The allocated computing time is greatfully acknowleged. Finally, the authors want to thank Swen Blobel, Dr. Eike Dohmen and Dr. Sven Werdin for the support in the production and testing of the polymer samples.

Author contribution: All the authors have accepted responsibility for the entire content of this submitted manuscript and approved submission.

Research funding: The present study is funded by the German Research Foundation, Priority Program (SPP) 1681 grants KA 3309/2-1/3. This support is gratefully acknowledged.

Conflict of interest statement: The authors declare no conflicts of interest regarding this article.

\section{References}

1. Becker TI, Böhm V, Vega JC, Odenbach S, Raikher YL, Zimmermann K. Magnetic-field-controlled mechanical behavior of magneto-sensitive elastomers in applications for actuator and sensor systems. Arch Appl Mech 2019;89:133-52.

2. Schubert G. Manufacture, characterisation and modelling of magneto-rheological elastomers. PhD thesis. University of Glasgow; 2014.

3. Tian TF, Li WH, Deng YM. Sensing capabilities of graphite based MR elastomers. Smart Mater Struct 2011;20:025022.

4. Volkova TI, Böhm V, Kaufhold T, Popp J, Becker F, Borin DY, et al. Motion behaviour of magnetosensitive elastomers controlled by an external magnetic field for sensor applications. J Magn Mater 2016;431:262-5.

5. Böse H, Rabindranath R, Ehrlich J. Soft magnetorheological elastomers as new actuators for valves. J Intell Mater Syst Struct 2012;23:989-94.

6. Carlson JD, Jolly MR. MR fluid, foam and elastomer devices. Mechatronics 2000;10:555-69.

7. Kim Y, Parada GA, Liu S, Zhao X. Ferromagnetic soft continuum robots. Sci Robot 2019;4:eaax7329.

8. Guðmundsson İ. A feasibility study of magnetorheological elastomers for a potential application in prosthetic devices. Master's thesis. University of Iceland; 2011.

9. Danas K, Kankanala SV, Triantafyllidis N.. Experiments and modeling of iron-particle-filled magnetorheological elastomers. J Mech Phys Solid 2012;60:120-38.

10. Ginder JM, Nichols ME, Elie LD, Tardiff JL. Magnetorheological elastomers: properties and applications. In MR Wuttig, editor, Smart structures and materials 1999: smart materials technologies. International Society for Optics and Photonics, SPIE; 1999, vol 3675, 131-138. 
11. Hiptmair F, Major Z, Haßlacher R, Hild S. Design and application of permanent magnet flux sources for mechanical testing of magnetoactive elastomers at variable field directions. Rev Sci Instrum 2015;86:085107.

12. Martin JE, Anderson RA, Williamson RL. Generating strange magnetic and dielectric interactions: classical molecules and particle foams. J Chem Phys 2003;118:1557-70.

13. Kalina KA, Brummund J, Metsch P, Kästner M, Borin DY, Linke JM, et al. Modeling of magnetic hystereses in soft mres filled with NdFeB particles. Smart Mater Struct 2017;26:105019-31.

14. Linke JM, Borin DY, Odenbach S. First-order reversal curve analysis of magnetoactive elastomers. RSC Adv 2016;6:100407-16.

15. Sánchez PA, Gundermann T, Dobroserdova A, Kantorovich SS, Odenbach S. Importance of matrix inelastic deformations in the initial response of magnetic elastomers. Soft Matter 2018;14: 2170-83.

16. Schümann M, Odenbach $\mathrm{S}$. The microstructure of magnetorheological materials characterized by means of computed X-ray microtomography. Phys Sci Rev 2019. Submitted.

17. Vaganov MV, Borin DY, Odenbach S, Raikher YL. Modeling the magnetomechanical behavior of a multigrain magnetic particle in an elastic environment. Soft Matter 2019;15:4947-60.

18. Spieler C, Kästner M, Goldmann J, Brummund J, Ulbricht V. XFEM modeling and homogenization of magnetoactive composites. Acta Mech 2013;224:2453-69.

19. Cremer P, Löwen H, Menzel AM. Tailoring superelasticity of soft magnetic materials. Appl Phys Lett 2015;107:171903.

20. Romeis D, Toshchevikov V, Saphiannikova M. Elongated micro-structures in magneto-sensitive elastomers: a dipolar mean field model. Soft Matter 2016;12:9364-76.

21. Romeis D, Metsch P, Kästner M, Saphiannikova M. Theoretical models for magneto-sensitive elastomers: a comparison between continuum and dipole approaches. Phys Rev 2017;95:042501.

22. Menzel AM. Mesoscopic characterization of magnetoelastic hybrid materials: magnetic gels and elastomers, their particle-scale description, and scale-bridging links. Arch Appl Mech 2019;89: 17-45.

23. Menzel AM, Löwen H. Modeling and theoretical description of magnetic hybrid materials bridging from meso- to macro-scales. Phys Sci Rev 2019. Submitted.

24. Biller AM, Stolbov OV, Raikher YL. Modeling of particle interactions in magnetorheological elastomers. J Appl Phys 2014;116:114904.

25. Galipeau E, Ponte Castañeda P. The effect of particle shape and distribution on the macroscopic behavior of magnetoelastic composites. Int I Solid Struct 2012;49:1-17.

26. Galipeau E, Ponte Castañeda P. A finite-strain constitutive model for magnetorheological elastomers: magnetic torques and fiber rotations. J Mech Phys Solid 2013;61:1065-90.

27. Javili A, Chatzigeorgiou G, Steinmann P. Computational homogenization in magneto-mechanics. Int J Solid Struct 2013;50:4197-216.

28. Kalina KA, Metsch P, Kästner M. Microscale modeling and simulation of magnetorheological elastomers at finite strains: a study on the influence of mechanical preloads. Int J Solid Struct 2016;102-103:286-96.

29. Metsch P, Kalina KA, Spieler C, Kästner M. A numerical study on magnetostrictive phenomena in magnetorheological elastomers. Comput Mater Sci 2016;124:364-74.

30. Metsch P, Kalina KA, Brummund J, Kästner M. Two- and three-dimensional modeling approaches in magneto-mechanics: a quantitative comparison. Arch Appl Mech 2018;89:47-62.

31. Rudykh S, Bertoldi K. Stability of anisotropic magnetorheological elastomers in finite deformations: a micromechanical approach. J Mech Phys Solid 2013;61:949-67.

32. Goshkoderia A, Rudykh S. Stability of magnetoactive composites with periodic microstructures undergoing finite strains in the presence of a magnetic field. Compos B Eng 2017;128:19-29. 
33. Chatzigeorgiou G, Javili A, Steinmann P. Unified magnetomechanical homogenization framework with application to magnetorheological elastomers. Math Mech Solid 2012;19:193-211.

34. Keip M-A, Rambausek $M$. A multiscale approach to the computational characterization of magnetorheological elastomers. Int J Numer Methods Eng 2016;107:338-60.

35. Keip M-A, Rambausek M. Computational and analytical investigations of shape effects in the experimental characterization of magnetorheological elastomers. Int J Solid Struct 2017;121:1-20.

36. Dorfmann A, Ogden RW. Nonlinear magnetoelastic deformations of elastomers. Acta Mech 2004; 167:13-28.

37. Bustamante R. Transversely isotropic nonlinear magneto-active elastomers. Acta Mech 2010;210: 183-214.

38. Kankanala SV, Triantafyllidis N. On finitely strained magnetorheological elastomers. J Mech Phys Solid 2004;52:2869-908.

39. Saxena P, Hossain M, Paul S. A theory of finite deformation magneto-viscoelasticity. Int I Solid Struct 2013;50:3886-97.

40. Saxena $P$, Hossain $M, P a u l ~ S$. Nonlinear magneto-viscoelasticity of transversally isotropic magneto-active polymers. Proc R Soc Lond Math Phys Eng Sci 2014;470:20140082.

41. Haldar K, Kiefer B, Menzel A. Finite element simulation of rate-dependent magneto-active polymer response. Smart Mater Struct 2016;25:104003.

42. Lefèvre $\mathrm{V}$, Danas $\mathrm{K}$, Lopez-Pamies $\mathrm{O}$. Two families of explicit models constructed from a homogenization solution for the magnetoelastic response of mres containing iron and ferrofluid particles. Int J Non Linear Mech 2020;119:103362.

43. Mukherjee D, Bodelot L, Danas K. Microstructurally-guided explicit continuum models for isotropic magnetorheological elastomers with iron particles. Int J Non Lin Mech 2019;120:103380.

44. Kalina KA, Metsch P, Brummund J, Kästner M. Development of a macro-model for magnetorheological elastomers based on microscopic simulations. Int J Solids Struct 2020; 193-194:200-12.

45. Gebhart P, Attaran A, Wallmersperger T. Multiphysics modeling of fluid-saturated porous ferrogels at finite strains. Phys Sci Rev 2019. Submitted.

46. Pössinger T. Experimental characterization, modeling and simulation of magneto-rheological elastomers. PhD thesis. Ecole Polytechnique; 2015.

47. Bodelot L, Voropaieff J-P, Pössinger T. Experimental investigation of the coupled magnetomechanical response in magnetorheological elastomers. Exp Mech 2018;58:207-21.

48. Jackson JD. Klassische elektrodynamik, 4 ed. Berlin: Walter de Gruyter; 2006.

49. Eringen AC, Maugin GA. Electrodynamics of continua I: foundations and solid media. New York: Springer; 1990.

50. de Groot SR, Suttorp LG. Foundations of electrodynamics. Amsterdam: North-Holland; 1972.

51. Stark S, Semenov AS, Herbert B. On the boundary conditions for the vector potential formulation in electrostatics. Int J Numer Methods Eng 2015;102:1704-32.

52. Coleman BD, Noll W. The thermodynamics of elastic materials with heat conduction and viscosity. Arch Ration Mech Anal 1963;13:167-78.

53. Hill R. Elastic properties of reinforced solids: some theoretical principles. J Mech Phys Solid 1963; 11:357-72.

54. Petra CG, Schenk O, Anitescu M. Real-time stochastic optimization of complex energy systems on high-performance computers. IEEE Comput Sci Eng 2014a;16:32-42.

55. Petra CG, Schenk O, Lubin M, Gärtner K. An augmented incomplete factorization approach for computing the schur complement in stochastic optimization. SIAM J Sci Comput 2014b;36: C139-62.

56. Alnæs M, Blechta J, Hake J, Johansson A, Kehlet B, Logg A, et al. The Fenics project version 1.5. Arch Numer Soft, 3, 2015. 
57. Logg A, Mardal K-A, Wells G. Automated solution of differential equations by the finite element method: the FEniCS book. Springer Science \& Business Media, 2012, vol 84.

58. Danas K. Effective response of classical, auxetic and chiral magnetoelastic materials by use of a new variational principle. J Mech Phys Solid 2017;105:25-53.

59. Ponte Castañeda P, Galipeau E. Homogenization-based constitutive models for magnetorheological elastomers at finite strain. J Mech Phys Solid 2011;59:194-215.

60. Anders B. Magnetic vector hysteresis model with dry friction-like pinning. Phys B Condens Matter 1997;233:342-7.

61. Jiles DC, Atherton DL. Theory of ferromagnetic hysteresis. J Magn Mater 1986;61:48-60.

62. Lubliner J. Plasticity theory. Courier Corporation; 2008.

63. Dohmen E. Zum Einsatz magnetischer Hybridmaterialien in adaptiven Leichtbaustrukturen. PhD thesis. TU Dresden; 2016.

64. Mullins L. Effect of stretching on the properties of rubber. Rubber Chem Technol 1948;21:281-300.

65. Flory PJ. Thermodynamic relations for high elastic materials. Trans Faraday Soc 1961;57:829-38.

66. Jolly MR, Carlson JD, Muñoz BC. A model of the behaviour of magnetorheological materials. Smart Mater Struct 1996a;5:607.

67. Jolly MR, Carlson JD, Muñoz BC, Bullions TA. The magnetoviscoelastic response of elastomer composites consisting of ferrous particles embedded in a polymer matrix. J Intell Mater Syst Struct $1996 \mathrm{~b} ; 7: 613-22$.

68. Puljiz M, Huang S, Kalina KA, Nowak J, Odenbach S, Kästner M, et al. Reversible magnetomechanical collapse: virtual touching and detachment of rigid inclusions in a soft elastic matrix. Soft Matter 2018;14:6809-21.

69. Kalina KA, Brummund J, Metsch P, Kästner M. Modeling and simulation of hysteresis effects in magnetorheological elastomers. Proc Appl Math Mech 2018;18:e201800319.

70. Terada K, Kato J, Hirayama N, Inugai T, Yamamoto K. A method of two-scale analysis with micromacro decoupling scheme: application to hyperelastic composite materials. Comput Mech 2013; 52:1199-219.

71. Bustamante R, Dorfmann A, Ogden RW. Numerical solution of finite geometry boundary-value problems in nonlinear magnetoelasticity. Int J Solid Struct 2011;48:874-83.

72. Gebhart $\mathrm{P}$, Wallmersperger T. A general framework for the modeling of porous ferrogels at finite strains. J Mech Phys Solid 2019;122:69-83.

73. Vogel F, Bustamante R, Paul S. On some mixed variational principles in magneto-elastostatics. Int J Non Linear Mech 2013;51:157-69.

74. Birster K, Schweitzer R, Tschöpe A. Magnetic field-induced deformations of ni-nanorod/hydrogel composites. Phys Sci Rev 2019. Submitted.

75. Weeber R, Kreissl P, Holm C. Studying the field-controlled change of shape and elasticity of magnetic gels using particle-based simulations. Arch Appl Mech 2018;89:3-16. 
